\title{
Modeling Mechanical Patterns for Striated Muscles
}

\author{
Valery B. Kokshenev \\ Departamento de Fisica, Instituto de Ciencias Exatas, Universidade Federal de Minas Gerais, \\ Belo Horizonte, Brazil \\ Email: valery.kokshenev@gmail.com
}

Received 9 April 2014; revised 25 May 2014; accepted 7 June 2014

Copyright (C) 2014 by author and Scientific Research Publishing Inc.

This work is licensed under the Creative Commons Attribution International License (CC BY). http://creativecommons.org/licenses/by/4.0/

(c) () D Den Access

\section{Abstract}

Muscles show a surprisingly large variety of functions when they mechanically respond to different environmental requests. However, the in vivo workloop studies distinguish well only four patterns of skeletal muscles, producing positive, negative, almost zero and zero net works, that qualifies them respectively as motors, brakes, struts, and springs. While much effort of comparative biologists has been done in searching for muscle design patterns, no fundamental concepts underlying such four primary patterns were established. In this interdisciplinary study, continuum mechanics is applied to the problem of muscle structure in relation to function. The known ability of a powering muscle as whole to be tuned via natural (resonant) frequency to the efficient locomotion is now modeled through the non-linear elastic muscle moduli, controlling both the contraction frequency and velocity. When incorporated in activated skeletal and cardiac (striated) muscles via the mechanical similarity between loaded and reaction forces, further exploration of elastic force patterns (borrowed from solid state physics) yields an explicit rationalization for currently known locomotor muscle patterns. Besides explanation of the origin of allometric exponents derived for leg muscles in animals adapted to fast running and wing muscles in flying birds, the skeletal and cardiac muscles are patterned through the primary and secondary high power activities. Further applications are expected to be useful in designing of artificial muscles and modeling living and extinct animals.

\section{Keywords}

Continuum Mechanics, Modeled Muscles, Workloops, Scaling Elastic Effects, Allometry, Active Stiffness 


\section{Introduction}

The mechanical role of muscles varies widely with their architecture and activation conditions. Striated (skeletal and cardiac) muscles are diverse in their contractive interspecific and intraspecific functional properties observed among and within animal species, nevertheless, in all cases "the smaller muscles and muscles of smaller animals are quicker” [1]. After Hill [1] who first noted this generic feature of the design of skeletal muscles, their physiological adaptation, resulting in beneficial changes in muscle function, has been recognized by a number of investigators. For example, it was learned that long-fibre muscles commonly contract at over larger length ranges and relatively higher velocities producing the greatest muscle forces the lowest relative energetic costs [2]. Muscles having shorter fibres expose smaller length change, but their cost of force generation is relatively less, e.g. [3]. Searching for determinants of evolution of shape, size, and force output of cardiac and skeletal muscle, a little is known about the regulation of directional processes of mass distribution [4] [5]. Although skeletal muscles grow in length as the bones grow, most studies only involve force increasing with respect to cross-sectional area. Following the idea that the muscle force production function is a critical evolutionary determinant [5], I develop a physical study of the muscle form adaptation to a certain primary activity with growth of size (length and cross-sectional area) under evident condition of the preservation of muscle shape.

When designing architecture of the striated muscle built from repeating units (fibres and sarcomeres) at least three distinct muscle activities should be distinguished [5]: the concentric contraction defined as the production of active tension while the muscle is shortening and performing positive work, the eccentric contraction defined as contraction during lengthening performing negative work in a controlled fashion, and the isometric contraction when the muscle force output is produced without changing of length and performing almost zero or zero net work. The corresponding mechanical work patterns called by Russel et al. [5] as "concentric work" and "eccentric work" (that might be extended here by “isometric work”) were carefully studied via in vivo measurements of length-force cycling (workloops) of individual skeletal muscles in active animals. Presented (in Figure 3 in [6]) by the pectoralis in flying birds, leg extensors in running cockroaches, gastrocnemius in the level running turkey, and intrinsic wing muscles in insects, the corresponding muscle locomotor patterns are known as the motor, brake, strut and spring muscles [6].

The seminal research by Hill [1] on dynamics of electrically stimulated isolated muscles was restricted to a single isotonic shortening. The studies of the relevant motor function resulted in famous force-inverse-velocity master curve presenting the major dynamic constraint of all real (slow-fibre, fast-fibre, and superfast) muscles [7] and computationally modeled muscles, e.g. [8]. Besides, other two fundamental rules of muscle dynamics were noted by Hill [1]. Examining hovering humming and sparrow birds, he recognized that the "frequencies of wings are roughly in inverse proportion to the cube roots of the weights, i.e. to linear size”. Moreover, because the linear proportionality between the stroke period $T$ and body length $L$ was equally established in in vivo and electrically stimulated isolated muscles, the corresponding frequency-inverse-length scaling rule $T_{m}^{-1} \propto L_{m}^{-1} \sim L^{-1}$, shown for a given muscle $m$, is likely more universal than previously appreciated and associated with the nervous control. Second velocity-inverse-length Hill's constraint states that "the intrinsic speed of muscle has to vary inversely to length”, i.e. $V_{m} \propto L_{m}^{-1}$. Both Hill's scaling rules still remain a challenge to viscoelastic models of transient-state muscle mechanics and other theories of muscle contraction, e.g. [9].

The earliest theories of muscle motor function supposed muscle to be an elastic body which, when stimulated, was converted in an active state containing elastic energy causing the muscle to shorten. Such elastic-energy theories failed to explain mechanisms of the force production in terms of viscoelastic characteristics. To a certain extent, poor experimental approaches providing often conflicting clues to muscle structure in relation to function may explain a little progress in understanding of contractile properties of a muscle [4] [9]. Moreover, physiological muscle properties accounted for macroscopic theories, i.e. (developed at macroscopic scale of consideration of muscle contractions, are primarily focused on the reproduction of force-velocity curve [9]. Besides, the existing phenomenological frameworks such as Hill-type muscle models only mimic the proper mechanical characteristics of muscles by means of passive viscoelastic springs attached to muscle contractive element in series [3] [10] [11] or in parallel [12] and recruited when muscle is activated. Such kind of models ignores the fact that the force production is followed by the process of force transmission throughout the same muscle organ. In other words, Hill-type muscle models employ Hill's principal constraints of muscle dynamics, not being able to explain them. In line with the experimental evidences of the adaptive ability of skeletal muscle to exchange elastic strain energy during force production [2], it was communicated on the force-similarity approach to modeling of the four primary muscle patterns via the adaptive muscle elasticity [13]. 
In the present paper, I develop an integrative theoretical approach to the problem of active forces, mechanically adapted design, and contractive linear and non-linear dynamics of striated muscles. Instead of Hill-type modeling of in vitro motor function (e.g. [3]), brake function (e.g. [2] [12]), and strut function (e.g. [14]), or study of muscle design by means of simulation of phenomenological force-length and/or force-velocity constraints [8], the powerful method of continuum mechanics generally providing macroscopic characterization and modeling of soft tissues (e.g. [15] [16]) is employed. As a further exploration of the elastic force patterns, I propose a self-consistent depiction of the three dynamically-distinct point characteristics of typical in vivo forcelength loops of the naturally activated skeletal muscles. Unlike the earliest elastic theories based on minimization of energy, I develop the physical concept of similarity between the force output and reaction active elastic forces that permits one to avoid the molecular-scale details of the muscle activation process. The theory is validated by a comparison to phenomenological scaling rules including both mentioned Hill's dynamic constraints and therefore may be hopefully helpful in designing artificial muscles [15] and modeling living and extinct organisms [17].

\section{Theory}

\subsection{Theoretical Background}

\subsubsection{McMahon's Scaling to Body Weight}

The engineering models by McMahon [18] [19] develop previous Hill's approach to the problem of scaling quantities of animal performance to body weight $W=M g$. Using Hill's geometric similarity models [1] [19] equally applied to animal body, long bone, or individual muscle, each one was approximated by a cylinder of longitudinal length $L$ and cross-sectional area $A$ (or diameter $D \sim \sqrt{A}$ ). Moreover, the assumption on the weight-invariance of for the tissue density was adopted, namely

$$
\rho_{\text {tiss }}=\frac{M}{A L} \propto W^{0} .
$$

In mammalian long-bone allometry, this invariant was verified and observed with a high precision [20]. Mechanical models of bending bones and shortening muscles were introduced by McMahon via the weight-invariant elastic modulus $E_{\text {tiss }}$, tissue stress $\sigma_{\text {tiss }}$ and strain $\varepsilon_{\text {tiss }}$, namely

$$
E_{\text {tiss }}=\frac{\sigma_{\text {tiss }}}{\varepsilon_{\text {tiss }}} \propto W^{0} \text {, with } \sigma_{\text {tiss }}=\frac{\Delta F}{A} \text { and } \varepsilon_{\text {tiss }}=\frac{\Delta L}{L} \text {. }
$$

Here $\Delta L\left(=L-L_{0}\right)$ is the length change accompanied by the force change $\Delta F\left(=F-F_{0}\right)$ counted off from the resting length $L_{0}$.

While searching for functional mechanical patterns of biological systems determined by maximal forces using Equation (1) and Equation (2), the maximal-amplitude stress/strain scaling relations

$$
\sigma_{\text {geom }}^{(\max )} \propto W^{1 / 3}, \sigma_{\text {elast }}^{(\max )} \propto W^{1 / 4} \text {, and } \sigma_{\text {stat }}^{(\max )} \propto W^{1 / 5},
$$

could be readily derived from McMahon's geometric (isometric elastic stress), elastic (buckling elastic stress) and static (bending elastic stress) similarity models distinguished through McMahon's scaling relations

$$
L_{\text {geom }} \sim D, L_{\text {elast }} \propto D^{2 / 3} \text {, and } L_{\text {stat }} \propto D^{1 / 2} .
$$

Instead, the maximum stress and strain

$$
\sigma_{\text {tiss }}^{(\max )} \propto \varepsilon_{\text {tiss }}^{(\max )} \propto W^{0},
$$

were postulated (in Table 4 in [19]) thereby groundlessness extending McMahon's exact result for the mean stress $\sigma_{\text {elast }}^{\text {(mean) }} \propto W^{0}$, obtained within the static stress similarity model (see Figure 1 in [19]). The improved self-consistent maximal stresses shown in Equation (3) follow straightforwardly from McMahon's cross-sectional areas

$$
A_{\text {geom }}^{(\text {isom })} \propto W^{2 / 3}, A_{\text {elast }}^{(\text {buck }} \propto W^{3 / 4} \text {, and } A_{\text {static }}^{(\text {bend })} \propto W^{4 / 5},
$$

when applied to Equation (2), along with McMahon's idea on the dominating role of gravitational forces in bones, muscles, and bodies, i.e. $\Delta F \sim g M_{b} \sim g M_{m} \sim W$. 
The patterns of long bones are generally driven by the peak muscle forces, but not by gravity, as repeatedly noted by many authors, proven [20] and exemplified by all mammals as whole [21]. Nevertheless, one amazing case of the experimental evidence of McMahon's elastic similarity is due to limb bones in African elephants which, in contrast to Asian elephants, are most likely adapted for axial bone compression, influenced by gravitational reaction forces [21].

Although the evolution of locomotor trends of terrestrial giants are likely driven by body weight [22], the idea on the origin of locomotion patterns of animals (running, flying, and swimming) based on minimization of useful energy in the gravitational field [23], was also confronted with the new idea of maximum body efficiency in the muscular field [24].

\subsubsection{Muscle Shape and Structure}

After Alexander [25], the physiologic cross-sectional area $A_{0 m}$ (PCSA) of the isolated skeletal muscle $m$ of mass $M_{m}$ composed of $N$ bundles of masses $m_{i}$ was commonly estimated, e.g. [26], with the help of the cylinder-geometry relation $A_{i}=m_{i} / \rho_{m} L_{i}$, where $\rho_{m}$ is the muscle density and $L_{i}$ is directly measured muscle fibre length. The spindle-like shape of the muscle as whole organ was therefore determined by the muscle PCSA, namely

$$
\text { since } A_{0 m}=\sum_{i=1}^{N} A_{i}=\frac{M_{m}}{\rho_{m} L_{0 m}} \text { and } M_{m}=\sum_{i=1}^{N} m_{i} \text {, hence } \frac{1}{L_{0 m}}=\frac{1}{M_{m}} \sum_{i=1}^{N} \frac{m_{i}}{L_{i}},
$$

As shown [25] [26], the sum of areas of the muscle and the muscle length $L_{0 m}$ of the parallel-linked contractible subunits is described statistically by the length-unversed sum weighed by masses. Such a simplified (coarse-grained) characterization of the muscle structure generally ignores the arrangement of muscle fibres relative to generated force axis, distinguished by pinnate angles.

In scaling models, the evolution of the muscle structures across different-sized animals of body mass $M$ is observed statistically via allometric exponents $a_{m}, l_{m}$, and $\alpha_{m}$ determined by common rules [25] [27] [29]:

$$
A_{0 m} \propto M_{m}^{a_{m}}, L_{0 m} \propto M_{m}^{l_{m}} \text {, and } M_{m} \propto M^{1+\alpha_{m}} \text {, }
$$

where the muscle mass index $\alpha_{m}$ plays the same role as Prangel's index $\beta$ in bones, as noted in [30]. When the muscle-density invariance employed implicitly in Equation (7) and specified in Equation (1) is applied to different skeletal muscles, the muscle shape approximated by cylinder geometry is also preserved. Consequently, the muscle functional volume

$$
A_{m} L_{m}=A_{0 m} L_{0 m}=\frac{M_{m}}{\rho_{0 m}}, \text { with } \rho_{0 m}=\rho_{m} \propto M_{m}^{0} \sim M^{0},
$$

holding in all muscle activities, plays the role of the muscle mechanical invariant. This statement is ensured by the functional variation of density $\Delta \rho_{m} / \rho_{m}$ not exceeding 5\% [28]. Hence, the function-independent muscle-shape constraint [13]

$$
a_{m}+l_{m}=1+\alpha_{m}
$$

straightforwardly follows from Equation (8) and Equation (9). Likewise the case of hindlimb mammalian bones of the mean structure $a_{b}^{(\exp )}=2 d_{b}^{(\exp )}=0.752, l_{b}^{(\exp )}=0.298$, and $\beta^{(\exp )}=0.04$ [20] [30], Equation (10) is also empirically observable in muscle allometry (see further analysis in Table 5).

\subsection{General Muscle Characterization}

\subsubsection{Maximal Force and Stress}

Using the in vivo workloops, the muscle locomotor patterns can be generally specified regardless of details of activation-deactivation conditions. In Figure 1, the linear-slope characteristics $L_{1 m}$ can be introduced in the force-length cycling by the length-point conditions: $L_{2 m}<L_{1 m}<L_{3 m} \approx L_{0 m}$, for the motor function, $L_{2 m}>L_{1 m}>L_{3 m}$, for the brake function, and by $L_{2 m} \gtrsim L_{1 m} \gtrsim L_{3 m} \approx L_{0 m}$, for the strut function showing nearly isometric muscle contractions.

Moreover, such a qualitative general characterization of the activated individual muscle $m$ of resting length $L_{0 m}$ can be rationalized on the basis of common two-point force-length description, namely

$$
F_{\text {musc }}^{(\exp )}\left(L_{2 m}\right)=F_{\text {musc }}^{(\max )}=F_{2 m} \text { and } F_{\text {musc }}^{(\exp )}\left(L_{1 m}\right)=F_{1 m} \text {, }
$$




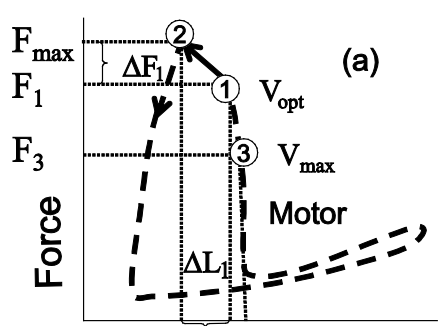

L $\quad \mathrm{L}_{1} \mathrm{~L}_{0} \quad$ Length

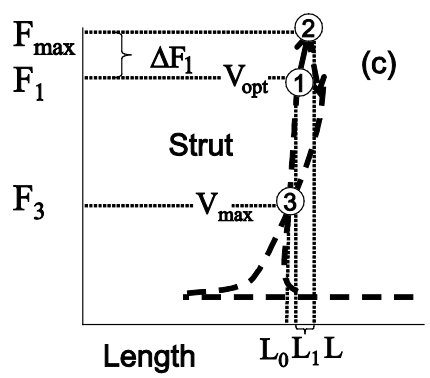

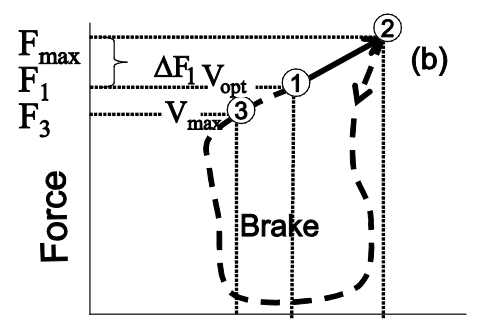

$\mathrm{L}_{0} \mathrm{~L}_{1}^{\Delta \mathrm{L}_{1}} \mathrm{~L}$

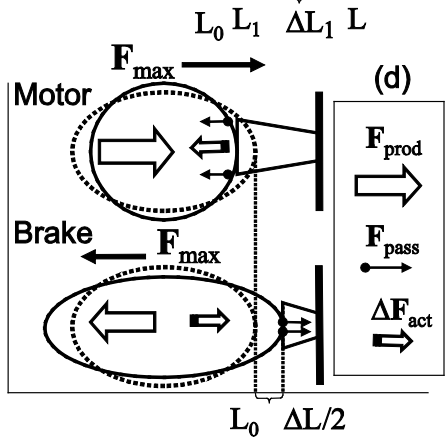

Figure 1. The qualitative analysis of the in vivo muscle force-length data. The muscle motor function is presented by gastrocnemius powering during shortening in uphill running turkey (inset a, adapted from [31]). The lateral gastrocnemius and plantaris act as brake (inset $b$ ) and strut (inset c) in hopping tammar wallabies [28]. The solid (and dashed) arrows indicate rasing (and decreasing) of the exerted force near its maximum magnitude $F_{\max }$, distinguished by the turning point 2 . The regions of the linear force-length domain are displayed by the force change $\Delta F_{1 m}$ and length change $\Delta L_{1 m}$, estimated from point 1 as the starting datapoint of the force enhancement $F_{1 m}$ with length $L_{1 m}$, achieved at the optimum contraction velocity $V_{o p t}$ and frequency. Similar to cyclic pendulum, the activated muscle is expected to pass though point 3 with maximum contraction velocity $V_{\max }$ at resting length $L_{0 m}$, with moderate force $F_{3 m}$. Inset $d$ : The resulted intrinsic force generated by the powering shortening (motor) or lengthening (brake) muscle, exemplified by $F_{\max }$, is due to a superposition of the production force output $F_{\text {prod }}$ and elastic forces, reaction passive $F_{\text {pass }}$ and active $F_{a c t}$ (see also text below Equation (16)).

introduced by the maximum force $F_{2 m}^{(\max )}$ and the optimum muscle length [32] [33] $L_{1 m}$. The instant dynamic length $L_{m}=L_{1 m} \pm \Delta L_{1 m}$ is counted off from the characteristic point $L_{1 m}$ via the optimum length change $\Delta L_{1 m}$ shown in Equation (11) and Figure 1 for all locomotory functions.

First, the linearization of the in vivo muscle force-length curve allows one to determine the trial peak stress and strain by

$$
\sigma_{\text {musc }}^{(\max )}=\frac{F_{\text {musc }}^{(\max )}}{A_{2 m}} \text { and } \varepsilon_{\text {musc }}^{(\max )}=\frac{\Delta L_{1 m}^{(\max )}}{L_{2 m}} \text {, with } \Delta L_{1 m}^{(\max )}=\left|L_{2 m}-L_{1 m}\right| .
$$

The corresponding force change $\Delta F_{\text {musc }}^{(\max )}$ observed near the optimum force $F_{1 m}^{(\max )}$ provides

$$
F_{\text {musc }}^{(\max )}=F_{\text {musc }}^{(\max )}\left(L_{1 m}\right)+\Delta F_{\text {musc }}^{(\max )}=F_{1 m}+K_{\text {musc }}^{(\max )} \Delta L_{1 m}^{(\max )}
$$

that in turn determinates effective muscle stiffness $K_{2 m}$ and effective modulus $E_{2 m}$, namely

$$
K_{\text {musc }}^{(\max )} \equiv K_{2 m}=\left|\frac{\mathrm{d} F_{\text {musc }}}{\mathrm{d} L_{m}}\right|_{F_{1 m}^{(\max )}} \approx \frac{\Delta F_{\text {musc }}^{(\max )}}{\Delta L_{1 m}^{(\max )}}=\frac{\Delta F_{\text {musc }}^{(\max )}}{F_{\text {musc }}^{(\max )}} E_{\text {musc }}^{(\max } \frac{A_{2 m}}{L_{2 m}} \text {, and } E_{\text {musc }}^{(\max )} \equiv E_{2 m}=\frac{\sigma_{\text {musc }}^{(\max )}}{\varepsilon_{\text {musc }}^{(\max )}},
$$


following from Equation (12) and Equation (13).

\subsubsection{Active Stiffness and Resonant Muscle Mechanics}

Secondly, treating the maximum-force crossover state as the generic transient-neutral state [30], the resonant frequency $1 / T_{\text {musc }}^{(\max )}=T_{2 m}^{-1}$ related to point 2 in Figure 1 and associated with maximum efficiency of muscle cycling, e.g. [34], can also be introduced as natural frequency [19] [34], namely

$$
T_{2 m}^{-1} \sim 2 \pi \sqrt{\frac{K_{2 m}}{M_{m}}} \sim \sqrt{\frac{E_{\text {musc }}^{(\max )}}{\rho_{0 m}}}\left(\frac{\Delta F_{\text {musc }}^{(\max )}}{F_{\text {musc }}^{(\max )}}\right)^{1 / 2} \frac{1}{L_{2 m}} \propto \frac{\sqrt{E_{\text {musc }}^{(\max )}}}{L_{2 m}} .
$$

One can see that Equation (15) yields first Hill's frequency-inverse-length constraint discussed in Introduction. However, the following three observation conditions of this constraint are required: 1) the preservation of dynamic functional volume (see Equation (9)), 2) the weight-invariance of the elastic modulus $E_{\text {musc }}^{(\max }$, and 3) the validation of force similarity between the exerted force $F_{\text {musc }}^{(\max )}$ and its change $\Delta F_{\text {musc }}^{(\max )}$ (see Equation (13)). Therefore, the muscle force-similarity principle, implying a coexistence of all forces in biomechanically equivalent states [30], can be formulated as

$$
F_{\text {musc }} \cong \Delta F_{\text {musc }} \cong F_{\text {prod }} \cong F_{\text {elast }} \cong \Delta F_{\text {elast }} .
$$

Here the active elastic force $\Delta F_{\text {elast }}$ (shown schematically in the inset d (see Figure 1 as $F_{\text {act }}$ ) is also included $^{1}$. The total transient-state elastic force $F_{\text {elast }}$ is the superposition of common passive elastic force $F_{\text {pass }}$ provoked by external loads and active elastic force $\Delta F_{\text {elast }}$ caused by the production force $F_{\text {prod }}$.

Given that the peak active muscle stress $\sigma_{m}$ always exceeds the corresponding passive stress, e.g. [14], in further I focus on transient states in the fully activated muscle (restricted by points 1 and 2 in Figure 1) and described by

$$
\sigma_{m}=\frac{\Delta F_{\text {elast }}}{A_{m}}=E_{m} \frac{\Delta L_{m}}{L_{m}} .
$$

Unlike the trial peak stress in Equation (12), $\sigma_{m}$ is the true intrinsic elastic stress in a certain, non-specified transient dynamic state. This reveals elastic force change near the maximum amplitude

$$
\Delta F_{\text {elast }} \equiv \Delta F_{m}=K_{m} \Delta L_{m}=E_{m} A_{m} \frac{\Delta L_{m}}{L_{m}}
$$

and in turn provides the corresponding active muscle stiffness

$$
K_{m}=E_{m} \frac{A_{m}}{L_{m}} .
$$

The underlying mechanical sarcomere elastic stiffness $K_{s}$ is related via the muscle-volume average, namely

$$
K_{m}=\frac{1}{A_{m} L_{m}} \int K_{s}\left(r_{m}\right) \mathrm{d}^{3} r_{m},
$$

originated from end-to-end intercellular overlapping [12] [35].

The muscle energy change

$$
\Delta U_{m} \sim K_{m} \Delta L_{m}^{2} \cong E_{m} A_{m} \frac{\Delta L_{m}^{2}}{L_{m}}
$$

stored or released during active-period contraction provides the mechanical cost of energy

$$
C U_{m}=\frac{\Delta U_{m}}{\Delta L_{m}} \cong E_{m} A_{m} \frac{\Delta L_{m}}{L_{m}} .
$$

These relations demonstrate how the observable mechanical characteristics can be linked to the underlying muscle elastic forces using the force-similarity principle formulated in Equation (16). In turn, the contraction

\footnotetext{
${ }^{1}$ The correspondence sign $\cong$ indicates that though the involved physical characteristics belong to the same mechanical state, they may dif-
} fer in both physical and numerical parameters stipulating this state. For other notations see Appendix A. 
velocity

$$
V_{m}=\overline{V_{m}(t)} \equiv \frac{1}{\Delta t_{m}} \int_{0}^{\Delta t_{m}}\left[\frac{\mathrm{d} L_{m}(t)}{\mathrm{d} t}\right] d t \sim\left[\frac{\mathrm{d} L_{m}(t)}{\mathrm{d} t}\right]_{t=\Delta t_{m}} \cong \frac{L_{m}}{T_{m}}
$$

is defined by the instant velocity $V_{m}(t)$ averaged over activation time $\Delta t_{m}$.

\subsubsection{Fast and Slow Activated Muscles}

According to the most general classification of diverse muscles, three types are conventionally distinguished: red (slow fibre) muscles, white (fast fibre) muscles, and intermediate type, mixed fibre muscles. Although collective mechanisms of muscle contractions are poor understood, e.g. [36], physically, two limiting situations of the dynamic accommodation of local forces generated by cross bridge attachments can be generally rationalized. As schematically drawn in the inset $d$ in Figure 1 for an activated muscle, the dynamic process of equilibration between the production intrinsic forces and external loads (not shown) is followed by the spatiotemporal relaxation of elastic forces. For the simplest case of slow muscles, the dynamic equilibration occurs via the slow channel of relaxation, supposedly common for both active, $F_{\text {prod }}^{(\text {slow }}$, and passive elastic forces. Since passive forces in solids are short of range [37], both the forces are proportional to muscle surface. In contrast, it is plausible to adopt that in fast muscles the fast-twitch fibres transmit the locally generated forces in all directions, i.e. along and across fibres, resulting in the overall maximum force output $F_{\text {prod }}^{(\text {fast }}$ to be linear with dynamic muscle volume. Basing on such a generalized physical picture, a function-independent and regime-independent characterization of the force production function, namely

$$
F_{\text {prod }}^{(\text {fast })} \propto A_{r m} L_{r m} \text { and } F_{\text {prod }}^{(\text {slow }} \propto A_{r m} \text {, with } r=1,2 \text {, and 3, }
$$

is proposed through the force-size scaling rules, for all three distinct states shown in Figure 1, hereafter distinguished by symbol $r$.

The linear-displacement regime with $\Delta L \sim L$ widely adopted among biologists for overall dynamic characterization, is discussed in Equation (2) that results in the weight-independent strain, that may shed light on stress postulated in Equation (5). The corresponding optimum-velocity regime $r=1$, attributed to the instant length-independent elastic strains, $\varepsilon_{m}^{(o p t)}=\left|L_{m}-L_{3 m}\right| / L_{m} \propto L_{m}^{0}$ with $L_{m}$ lying between $L_{1 m}$ and $L_{3 m} \approx L_{0 m}$, is now introduced by scaling equations

$$
E_{1 m}^{(\text {fast })}=E_{\text {fast }}^{(\text {opt })} \propto L_{m}^{1}, E_{1 m}^{(\text {slow })}=E_{\text {slow }}^{(o p t)} \propto L_{m}^{0}, \text { with } \Delta L_{1 m} \sim L_{m},
$$

characteristic of the fast and slow muscles. Such a muscle description follows from the principle of similarity (see Equation (16)) between the active elastic force $\Delta F_{1 m}=\Delta F_{\text {elast }}^{(\text {opt }}=E_{1 m} A_{1 m} \varepsilon_{1 m}$ (see Equation (18)) and corresponding production force (see Equation (24)). The optimum-force and optimum-velocity muscle mechanics is rationalized below in Table 1 and then tested by empirical data.

Likewise, the bilinear-displacement moderate-velocity regime $r=2$ introduced by the dynamic length change $\Delta L_{2 m}=\left|L_{m}-L_{1 m}\right| \propto L_{m}^{2}$, with $L_{m}$ lying between $L_{2 m}$ and $L_{1 m}$, and the maximum active elastic force $\Delta F_{2 m}=\Delta F_{\text {elast }}^{(\max }=E_{2 m} A_{2 m} \varepsilon_{2 m}^{(\max )}$ (see Equation (18)) results in the maximal elastic moduli

$$
E_{\text {fast }}^{(\max )}=E_{2 m}^{(\text {fast })} \propto L_{m}^{0}, E_{\text {slow }}^{(\max )}=E_{2 m}^{(\text {slow })} \propto L_{m}^{-1}, \text { with } \Delta L_{2 m} \propto L_{m}^{2},
$$

adjusted with the muscle production function on the basis of force similarity principle. Finally, the trilinear high-velocity regime $r=3$ is suggested by the moderate elastic muscle force determined by

$$
E_{\text {fast }}^{(\text {mod })}=E_{3 m}^{(\text {fast })} \propto L_{m}^{-1}, E_{\text {slow }}^{(\text {mod })}=E_{3 m}^{(\text {slow })} \propto L_{m}^{-2} \text {, with } \Delta L_{3 m} \propto L_{m}^{3} .
$$

This condition specifies point 3 in Figure 1, along with the underlying cubic-power muscle displacements $\Delta L_{3 m}$ scaled by dynamic $L_{m}$ lying above or below the characteristic length $L_{3 m}$ in any muscle acting as motor, brake or strut.

\subsection{Muscle Functions}

Likewise the naturally curved mammalian long bones biomechanically adapted to the maximum longitudinally bending [20] [30], the muscle motor function is assigned to locomotor muscles showing concentric positive 
work exerted by elastic bending forces. Given that the elastic force patterns coincide for bending and torsion [30], both kinds of unpinnate and uni-pinnate skeletal muscles, having respectively close to zero and non-zero fixed pinnate angles, may be expected to be structured by the same motor function. The specific-function mechanical characterization is described in Appendix $\mathbf{B}$ and results are summarized in Table 2.

Table 1. General mechanical characteristics of the striated muscles tuned to linear-displacement dynamic regime scaled to dynamic fibre length $L_{m}=L_{1 m}$. The mixed-fibre scaling dynamic exponents (shown in the last column) are modeled by the common means for the fast-muscle and slow-muscle exponents (established in the second and third columns), following from the rule $F_{\text {mix }} \sim \sqrt{F_{\text {fass }} F_{\text {slow }}} ; A_{m}$ and $L_{m}$ are attributed to the stabilized dynamic muscle geometry constrained by muscle volume Equation (9).

\begin{tabular}{lccc}
\hline \multicolumn{1}{c}{ Optimum muscle characteristics, Equation } & Fast fibres & Slow fibres & Mixed fibres \\
\hline Optimum length change, $\Delta L_{1 m}$, Equation (25) & $L_{m}$ & $L_{m}$ & $L_{m}$ \\
Production force, $\Delta F_{1 m}$, Equation (16), Equation (18), Equation (25) & $A_{m} L_{m}$ & $A_{m}$ & $A_{m} L_{m}^{1 / 2}$ \\
Optimum stiffness, $K_{1 m}=E_{1 m} A_{1 m} / L_{1 m}$, Equation (19) & $A_{m}$ & $A_{m} L_{m}^{-1}$ & $A_{m} L_{m}^{-1 / 2}$ \\
Optimum elastic stress, $\sigma_{1 m}=\Delta F_{1 m} / A_{1 m}$, Equation (17) & $L_{m}$ & $L_{m}^{0}$ & $L_{m}^{1 / 2}$ \\
Contraction frequency, $T_{1 m}^{-1} \sim \sqrt{E_{1 m} / \rho_{0 m}} / L_{1 m}$, Equation (15) & $L_{m}^{-1 / 2}$ & $L_{m}^{-1}$ & $L_{m}^{-3 / 4}$ \\
Optimum velocity, $V_{1 m}=V_{m u s c}^{(o p)}$, Equation (23) & $L_{m}^{1 / 2}$ & $L_{m}^{0}$ & $L_{m}^{1 / 4}$ \\
Optimum power, $P_{1 m}=F_{1 m} V_{1 m}$ & $A_{m} L_{m}^{3 / 2}$ & $A_{m}$ & $A_{m} I_{m}^{3 / 4}$ \\
\hline
\end{tabular}

Table 2. Scaling to mass of mechanical characteristics of muscles adapted to different locomotor functions. The allometric exponents related to animal's body mass (via Equation (8)) are presented in terms of muscle mass index $\alpha_{m}$. The powering individual muscles $m=1,2,3$, and 5 are tuned to the maximum-force bilinear dynamic regime $r=2$ (described in Equation (26)) and the control muscle $m=4$ acts in the linear regime $r=1$ (Equation (25)). *) The data shown only for the fast-fibre muscles. Other data are equally applied to fast, slow and mixed-fibre muscles.

\begin{tabular}{|c|c|c|c|c|c|}
\hline $\begin{array}{l}\text { Muscle pattern, regime } \\
\text { Equation }\end{array}$ & $\begin{array}{c}\text { Motor, } r=2 \\
\text { Equation (37) }\end{array}$ & $\begin{array}{c}\text { Brake, } r=2 \\
\text { Equation (41) }\end{array}$ & $\begin{array}{c}\text { Strut, } r=2 \\
\text { Equation (43) }\end{array}$ & $\begin{array}{l}\text { Control, } r=1 \\
\text { Equation (47) }\end{array}$ & $\begin{array}{c}\text { Pump, } r=2 \\
\text { Equation (45) }\end{array}$ \\
\hline $\begin{array}{l}\text { Force pattern, muscle } \\
\text { Equation }\end{array}$ & $\begin{array}{l}F_{\text {moror }}^{(\text {conc }}, m=1 \\
\text { Equation (35) }\end{array}$ & $\begin{array}{l}F_{\text {brocele }}^{\text {(ecce })}, m=2 \\
\text { Equation (39) }\end{array}$ & $\begin{array}{l}F_{\text {strut }}^{(\text {isom) }}, m=3 \\
\text { Equation (42) }\end{array}$ & $\begin{array}{l}F_{\text {conr }}^{\text {(spri) }}, m=4 \\
\text { Equation (46) }\end{array}$ & $\begin{array}{r}F_{\text {pump }}^{(\text {card })}, m=5 \\
\text { Equation (44) }\end{array}$ \\
\hline Maximum force, Equation (24) & $1+\alpha_{1}$ & $1+\alpha_{2}$ & $1+\alpha_{3}$ & $\frac{2}{3}\left(1+\alpha_{4}\right)$ & $1+\alpha_{5}$ \\
\hline Muscle fibre length, Equation (8) & $\frac{1}{5}\left(1+\alpha_{1}\right)$ & $\frac{1}{4}\left(1+\alpha_{2}\right)$ & 0 & $\frac{1}{3}\left(1+\alpha_{4}\right)$ & $\frac{1}{2}\left(1+\alpha_{5}\right)$ \\
\hline Cross-sectional area, Equation (8) & $\frac{4}{5}\left(1+\alpha_{1}\right)$ & $\frac{3}{4}\left(1+\alpha_{2}\right)$ & $1+\alpha_{3}$ & $\frac{2}{3}\left(1+\alpha_{4}\right)$ & $\frac{1}{2}\left(1+\alpha_{5}\right)$ \\
\hline Structure parameter, $\quad \eta_{m}=a_{m} l_{m}^{-1}$ & 4 & 3 & $\infty$ & 2 & 1 \\
\hline Length change"), Equation (26) & $\frac{2}{5}\left(1+\alpha_{1}\right)$ & $\frac{1}{2}\left(1+\alpha_{2}\right)$ & 0 & $\frac{1}{3}\left(1+\alpha_{4}\right)$ & $1+\alpha_{5}$ \\
\hline Maximum stress"), Equation (12) & $\frac{1}{5}\left(1+\alpha_{1}\right)$ & $\frac{1}{4}\left(1+\alpha_{2}\right)$ & 0 & 0 & $\frac{1}{2}\left(1+\alpha_{5}\right)$ \\
\hline Maximum stiffness ${ }^{*}$, Equation (19) & $\frac{3}{5}\left(1+\alpha_{1}\right)$ & $\frac{1}{2}\left(1+\alpha_{2}\right)$ & $1+\alpha_{3}$ & $\frac{1}{3}\left(1+\alpha_{4}\right)$ & 0 \\
\hline Natural frequency ${ }^{*}$, Equation (15) & $-\frac{1}{5}\left(1+\alpha_{1}\right)$ & $-\frac{1}{4}\left(1+\alpha_{2}\right)$ & 0 & $-\frac{1}{3}\left(1+\alpha_{4}\right)$ & $-\frac{1}{2}\left(1+\alpha_{5}\right)$ \\
\hline Energy change"), Equation (21) & $\frac{7}{5}\left(1+\alpha_{1}\right)$ & $\frac{3}{2}\left(1+\alpha_{2}\right)$ & $1+\alpha_{3}$ & $1+\alpha_{4}$ & $2\left(1+\alpha_{5}\right)$ \\
\hline Moderate velocity ${ }^{*}$, Equation (23) & 0 & 0 & 0 & 0 & 0 \\
\hline
\end{tabular}




\section{Results}

\section{Assumptions and Predictions}

The following assumptions are made regarding elastic striated muscles in fully activated states:

1) The powering individual muscles considered at macroscopic scale are treated as regime-dependent homogeneous solid-like organs. Within the scope of continuum medium mechanics, the macroscopic coarse-grained description ignores details of heterogeneous microstructure, including those resulting in pinnate angles.

2) When activated under different boundary loading conditions, the muscles do not undergo changes in shape and whole volume. The emerging muscular active-force fields [24] [38] follow the same patterns as passive elastic-force fields known in continuum medium mechanics of solids.

3) The mechanical similarity between the extrinsic forces exerted by the muscle and intrinsic elastic reaction forces, established above as the observation condition, can provide dynamic similarity features (for contraction velocities and frequencies), which can be theoretically and experimentally observable, at least in biomechanically equivalent states.

4) The natural ability of the non-linear elastic tuning of fast and slow muscles [34] can be characterized by the regime-dependent elastic moduli sensitive to evolving dynamic observable characteristics, e.g. the muscle length change.

One can deduce from Table 1, that the mechanical characterization of slow, fast and mixed muscles attributed to the linear-displacement regime $(r=1)$ is shape-dependent.

In Table 2, the scaling rules driving mass distribution in a given muscle $m$ are provided in terms of the muscle mass index $\alpha_{m}$. For example, the solution for the muscle-fibre length scaling exponent $l_{m}=\left(1+\alpha_{m}\right) / 5$ specified for the motor function $(m=1)$ follows from the muscle scaling Equation (8) and muscle motor pattern in Equation (37), both indicated in Table 2.

In Table 3, the scaling rules shown in Table 2 are compared with those for the optimal-force state related to the exponents $\left[a_{1 m}, l_{1 m}\right]$ and moderate-force state related to $\left[a_{3 m}, l_{3 m}\right]$. The dynamic characteristics of distinct-velocity contractions are predicted in Table 4.

Table 3. Locomotor functions predicted by dynamic structured for slow and fast striated muscles tuned to distinct dynamic regimes. The primary functions $(r=2)$ are shown by bold exponents. The analysis of functional muscle structures made in terms of elastic-force patterns: the active-muscle optimum-velocity $(r=1)$, moderate-velocity $(r=2)$, and high-velocity $(r=3)$ dynamic regimes are described in the first column via the muscle elastic moduli $E_{r m}$ (see Equations (25)-(27)) and specified by slow and fast force output (Equation (24)), shown in the second column. The third and next odd columns show the elastic force functional scaling in concentric, eccentric, isometric, and pump contractions. Unlike Table 2, the corresponding solutions to scaling equations underlaid by the force similarity principle Equation (16) are shown for simplicity with $\alpha_{r m}=0$. Notation: $n c$ indicates non-conclusive solution.

\begin{tabular}{|c|c|c|c|c|c|c|c|c|c|c|c|c|c|c|}
\hline & \multirow{2}{*}{$\begin{array}{c}\text { Dyn. regimes } \\
\qquad r=1,2,3\end{array}$} & \multirow{2}{*}{$\begin{array}{l}\text { Force } \\
F_{\text {prod }}^{\max } \propto\end{array}$} & \multirow{2}{*}{$\begin{array}{l}\text { Funct. } \\
F_{\text {motor }}^{\text {conc }} \propto\end{array}$} & \multicolumn{2}{|c|}{ Structure } & \multirow{2}{*}{$\begin{array}{l}\text { Funct. } \\
F_{\text {brake }}^{\text {eccen }} \propto\end{array}$} & \multicolumn{2}{|c|}{ Structure } & \multirow{2}{*}{$\begin{array}{l}\text { Funct. } \\
F_{\text {strut }}^{\text {isom }} \propto\end{array}$} & \multicolumn{2}{|c|}{ Structure } & \multirow{2}{*}{$\begin{array}{l}\text { Funct. } \\
F_{\text {pump }}^{\text {card }} \propto\end{array}$} & \multicolumn{2}{|c|}{ Structure } \\
\hline & & & & $a_{m}$ & $l_{m}$ & & $a_{m}$ & $l_{m}$ & & $a_{m}$ & $l_{m}$ & & $a_{m}$ & $l_{m}$ \\
\hline 1 & $E_{1 m}^{(s l o w)} \propto L_{m}^{0}$ & $A_{m}$ & $A_{m}^{\frac{3}{2}} L_{m}^{-1}$ & $\frac{2}{3}$ & $\frac{1}{3}$ & $A_{m}^{2} L_{m}^{-2}$ & $\frac{2}{3}$ & $\frac{1}{3}$ & $A_{m}$ & $n c$ & $n c$ & $L_{m}^{2}$ & $\frac{2}{3}$ & $\frac{1}{3}$ \\
\hline 2 & $E_{1 m}^{(f a s t)} \propto L_{m}$ & $A_{m} L_{m}$ & $A_{m}^{\frac{3}{2}}$ & $\frac{2}{3}$ & $\frac{1}{3}$ & $A_{m}^{2} L_{m}^{-1}$ & $\frac{2}{3}$ & $\frac{1}{3}$ & $A_{m} L_{m}$ & $n c$ & $n c$ & $L_{m}^{3}$ & $\frac{2}{3}$ & $\frac{1}{3}$ \\
\hline 3 & $E_{2 m}^{(s \text { low })} \propto L_{m}^{-1}$ & $A_{m}$ & $A_{m}^{\frac{3}{2}} L_{m}^{-2}$ & $\frac{4}{5}$ & $\frac{1}{5}$ & $A_{m}^{2} L_{m}^{-3}$ & $\frac{3}{4}$ & $\frac{1}{4}$ & $L_{m}^{-1} A_{m}$ & 1 & $\mathbf{0}$ & $L_{m}^{1}$ & $\frac{1}{2}$ & $\frac{1}{2}$ \\
\hline 4 & $E_{2 m}^{(\text {fast })} \propto L_{m}^{0}$ & $A_{m} L_{m}$ & $A_{m}^{\frac{3}{2}} L_{m}^{-1}$ & $\frac{4}{5}$ & $\frac{1}{5}$ & $A_{m}^{2} L_{m}^{-2}$ & $\frac{3}{4}$ & $\frac{1}{4}$ & $A_{m}$ & 1 & $\mathbf{0}$ & $L_{m}^{2}$ & $\frac{1}{2}$ & $\frac{1}{2}$ \\
\hline 5 & $E_{3 m}^{(s l o w)} \propto L_{m}^{-2}$ & $A_{m}$ & $A_{m}^{\frac{3}{2}} L_{m}^{-3}$ & $\frac{6}{7}$ & $\frac{1}{7}$ & $A_{m}^{2} L_{m}^{-4}$ & $\frac{4}{5}$ & $\frac{1}{5}$ & $L_{m}^{-2} A_{m}$ & 1 & 0 & $L_{m}^{0}$ & 0 & 1 \\
\hline 6 & $E_{3 m}^{(f a s t)} \propto L_{m}^{-1}$ & $A_{m} L_{m}$ & $A_{m}^{\frac{3}{2}} L_{m}^{-2}$ & $\frac{6}{7}$ & $\frac{1}{7}$ & $A_{m}^{2} L_{m}^{-3}$ & $\frac{4}{5}$ & $\frac{1}{5}$ & $A_{m} L_{m}^{-1}$ & 1 & 0 & $L_{m}^{1}$ & 0 & 1 \\
\hline
\end{tabular}


Table 4. Dynamic characterization of the red (slow) and white (fast) striated muscles in the optimum-, moderate-, and maximum-velocity dynamic regimes $r=1,2$, and 3 described in Table 3. Predictions are made on the basis of equations indicated in the table generalized over different dynamic regimes.

\begin{tabular}{cccccccc}
\hline Dynamic regime & Optimum & $(r=1)$ & Moderate & $(r=2)$ & Maximum & $(r=3)$ \\
\hline Muscle type & slow & fast & slow & fast & slow & fast \\
\hline Natural frequency, Equation (15), $T_{r m}^{-1} \propto$ & $L_{m}^{-1}$ & $L_{m}^{-1 / 2}$ & $L_{m}^{-3 / 2}$ & $L_{m}^{-1}$ & $L_{m}^{-2}$ & $L_{m}^{-3 / 2}$ \\
Contraction velocity, Equation (23), $V_{r m} \propto$ & $L_{m}^{0}$ & $L_{m}^{1 / 2}$ & $L_{m}^{-1 / 2}$ & $L_{m}^{0}$ & $L_{m}^{-1}$ & $L_{m}^{-1 / 2}$ \\
\hline
\end{tabular}

Some consequences of the proposed muscle scaling dynamic theory are:

1) The peak forces generated in all regimes scales as muscle volume or PCSA, respectively, in fast or slow muscles.

2) A general, function-independent mechanical description of the striated muscle activated in the liner-displacement regime is predicted for each type of muscles (Table 1).

3) The muscle-type independent locomotor functions and related mechanical and dynamic characteristics of the striated muscle activated in the bilinear regime are predicted (Table 2).

4) The muscle-type independent varied dynamic structures are predicted for all muscle regimes and functions (Table 3).

5) The function-independent dynamic scaling characteristics are obtained in Table 4 for all type of muscles.

In what follows, all theoretical findings are tested by available from the literature data.

\section{Analysis and Discussion}

Aiming to shed light on some important problems in the field of muscle dynamics, let me cite Louis Sullivan quoted in [5]: “What determines the shape, size, and force output of cardiac and skeletal muscle?”. Broadly, within the provided coarse-grained description of conservative striated muscles, the proposed theory suggests that the size-dependent peak elastic forces, emerging during the force production preserving muscle shape, are responsible for the muscle patterns observed via the functionally adapted structures. Moreover, the peak force output, which is described through the corresponding elastic force, is therefore determined by the muscle volume and cross-sectional area, respectively for white and red muscles, regardless of muscle functional specialization.

\subsection{Function against Structure}

\subsubsection{General Muscle Characterization}

Being composed of bundles of muscle fibres and other contractible components (neural, vascular, and collagenous reticulum), the striated muscle is thought of as a heterogeneous continuum medium transmitting the produced tension internally and externally, e.g. [39]. Primarily, I address the problem of mechanical design of striated muscle to a general, function-independent characterization of the individual muscle organ loaded by tension, reaction, and gravity through tendons, ligaments, and bones. My non-energetic approach is physically grounded by the existence of linear force-length regions (shown by the solid arrows in the workloops in Figure 1) revealed in all in vivo workloops regardless of dynamic details of approaching to the peak exerted force $F_{\text {musc }}^{(\max )}$. Hence, the mechanical characterization of the maximum-force activated muscle arises from the muscle stiffness $K_{m}^{(\max )}$ underlaid by sarcomere stiffness $K_{s}^{(\max )}$ discussed in Equation (20). Consequently, all forces involved in muscle contraction following by active and passive elastic strains allow common mechanical description (shown in the inset d in Figure 1) not depending on their biochemical, inertial, or reaction origin.

The analytical justification of Hill's frequency-inverse-length constraint results from the analysis of Equation (15) that eventually requires the usage of the similarity between all intrinsic muscle forces, Equation (16). The constraint $T_{m}^{-1} \propto L_{m}^{-1}$ and other mechanical characteristics for slow muscles shown in Table 1 can be generally applied to steady-speed regimes of locomotion modes where all forces are generally equilibrated and controlled by slow-fibre muscles [40]. In the case of non-steady transient locomotion when fast-twitch fibres and nervous control are additionally requested [40], Hill's first constraint transforms (by Equation (15) and Equation (25)) into a new one, $T_{m}^{-1} \propto L_{m}^{-1 / 2} \propto 1 / V_{\text {fast }}^{(o p t)} \quad$ (Table 1 and Table 4), well known for animals running with the maxim- 
al optimum speeds [41] [42] $V_{\text {run }}^{(\max )} \sim V_{\text {fast }}^{(\text {opt })} \propto \sqrt{L} \sim \sqrt{L_{m}}$.

Thereby, it has been demonstrated that the dynamic similarity establishes a link between the body-propulsion speed and locomotor-muscle contraction velocity, earlier described by Rome et al. [43]. Being united with the muscle-force similarity, both constraints lead to the mechanical similarity, as the basic principle explored in this research. More general approach to the problem of dynamic similarity in animal locomotion shows that the concept of mechanical similarity [24] and obtained above findings naturally follow from the key principle of analytical mechanics applied to the resonant (in frequency and phase) efficient cyclic locomotion [38].

\subsubsection{Maximum Force Output against Structure and Velocity}

In muscle physiology, the functional effect of muscle conceptual architecture simply states that muscle force output is proportional to PCSA. It may seem that the proposed study of the adaptation of muscle structure via force production function is in qualitative agreement with this statement, because in both cases of fast and slow muscles exposed in Equation (24) the muscle force output is proportional to $A_{m}$. Since a simplified treatment of the fast-muscle mechanics (in fact excluding other important dynamic variable $L_{m}$ ) leads to a widely adopted opinion that the size-independent peak stress $F_{\text {prod }}^{(\text {exp })} / A_{m}$, validating for the particular case of slow muscles (Table 1), is generic for all types of muscle, as already discussed in Equation (5).

Although the proposal on scaling of the maximum production force (and active stress) with muscle size shown in Equation (24) is a challenge for further research, the provided fairly general physical grounds are supported by empirical observations by Marden and Allen [44]. They statistically established that the peak force output in all biological (and human-made) motors falls into two fundamental scaling laws: 1) in fast-cycling motors, presented by flying insects, bats and birds, swimming fishes, and running animals the peak force scales as (motor mass) ${ }^{1}$ and 2) in slow-cycling motors, such as myosin molecules, muscle cells, and some (unspecified) "whole muscles" the force output scales as (motor mass) ${ }^{2 / 3}$; where the role of "motor mass" plays muscle (like fuel) mass. Within this context, the studied individual muscles are treated as complex biological motors, working due to actomyosin linkages (cross bridges), interacting in both longitudinal and transverse directions [5] [13]. The fact that muscle motors were observed from sarcomere to whole muscle organ passing through the single-fibre level of muscle organization, makes a basis for the introduced below micro-macro scale correspondence.

The study of the in vivo force-length curves is provided here in terms of the three distinct characteristic points (shown in Figure 1) characterized by the force and velocity inequalities

$$
F_{2 m}>F_{1 m}>F_{3 m} \text { and } V_{2 m}<V_{1 m}<V_{3 m} \text {. }
$$

These three function-independent generic states are associated with the linear $(r=1)$, bilinear $(r=2)$, and trilinear $(r=3)$ muscle dynamics determined via the muscle elastic moduli $E_{r m}$ in Equations (25)-(27), respectively. The mechanical characterization of slow and fast striated muscles is therefore provided in terms of the maximum $\left(\Delta F_{2 m}\right)$, optimum $\left(\Delta F_{1 m}\right)$ and moderate $\left(\Delta F_{3 m}\right)$ active elastic-force changes developed at the measurable maximum $\left(V_{3 m}\right)$, optimum $\left(V_{1 m}\right)$, and moderate $\left(V_{2 m}\right)$ contraction velocities (Table 4). The stabilization of the generic dynamic states is expected to be ensured by muscle tuning to natural frequencies, scaled to the dynamic length $L_{r m}$ and shown in Table 4 .

\subsubsection{Muscle Functions against Size and Shape}

Searching for answer on "what features make a muscular system well-adapted to a specific function?" [32], it has been communicated [13] that the natural conditions of stabilization of the moderate-velocity regime $r=2$, adjusted via the invariable fast-twitch fibre elastic moduli described here in Equation (26), result in muscle specific primary functions directly observed through the adapted resting muscle length (see Figure 1 in [13]).

Likewise [13], the elastic-force patterns, underlying concentric, eccentric, isometric, and cardiac contractions and determining eventually specific functions, are suggested, respectively in Equation (35), Equation (39), Equation (42), and Equation (44). The solutions to the muscle-force and muscle-shape constraints are accumulated in Table 2 as patterned functions well distinguished through the muscle structure parameter

$\left(\eta_{m}=\mathrm{d} \ln A_{m} / \mathrm{d} \ln L_{m}\right)$ established for such specialized skeletal muscles as the motor $\left(\eta_{1}=4\right)$, brake $\left(\eta_{2}=3\right)$, and strut $\left(\eta_{3}=\infty\right)$. They are now extended by the spring $\left(\eta_{4}=2\right)$ and pump $\left(\eta_{5}=1\right)$ for, respectively cardiac muscles and striated muscles. These structurally adapted muscles distinguished in Table 2 by index $m=1,2, \cdots, 5$ are thought of as to be suited to the efficient work when powering at resonant frequencies 
[34] during shortening ( $m=1, \eta_{1}=4$ ), lengthening ( $m=2, \eta_{2}=3$ ), or oscillating near the "isometric" dynamic state $\left(m=3, \eta_{3}=\infty\right)$, energy-saving state $\left(m=4, \eta_{4}=2\right)$, and high-pressure-resistant state $\left(m=5, \eta_{5}=1\right)$. The established lower structure parameter for the cardiac muscle $\left(\eta_{5}<\eta_{m}\right.$, with $\left.m \neq 5\right)$ is in accord with the description by Russel et al. [5] that "the heart chamber, unlike skeletal muscles, can extend in both longitudinal and transverse directions, and cardiac cells can grow in length and width". The scaling finding $\mathrm{d} \ln L_{5}=\mathrm{d} \ln A_{5}$ clearly indicates that the pump muscles may grow equally with mass in both along- and cross-sectional directions. Within this context, the scaling analysis tells us that the locomotor skeletal muscles are expected to grow more in length than width.

In Table 3, conceivable stable dynamic structures corresponding to muscle activity in different dynamic regimes are analyzed. As in the case of Table 2, the solutions to dynamic constraints follow from the similarity between the force output Equation (16) and elastic-force patterns. The resulting dynamic states are considered in terms of the scaling exponents for the muscle dynamic structure $\left[A_{r m}, L_{r m}\right]$ preserving muscle shape and volume Equation (9). Other related observable mechanical characteristics are exemplified in Table 1 and Table 2. The major outcome of the analysis in Table 3 is that both slow-twitch and fast-twitch fibres belonging to the same muscle $m$ should manifest concerted behavior coordinated by the dynamic active elastic forces and controlled by dynamic structure. As example, let us consider dynamic structure of the motor muscle $(m=1$ in Table 2) with the resting structure $\left[a_{0 m}=4 / 5, l_{0 m}=1 / 5\right]$ presented by item 4 in Table 3 . In the linear-displacement regime $r=1$, the fast motor preserves the dynamic structure $[2 / 3,1 / 3]$, that suggests the controllable spring as a secondary function for the motor. Likewise, the brakes and struts tuned to the linearly regime via the cycling frequency $T_{1 \text { slow }}^{-1}$ or $T_{1 \text { fast }}^{-1}$ (items 1 and 2, also described in Table 4) show the same multifunctional spring-type activity [13], as the secondary function. It is remarkable that the fast motors, brakes and struts being switched to the slow bilinear regime via $T_{2 \text { slow }}^{-1}$ work as slow motors, brakes and struts preserving the same corresponding dynamic structures (shown in item 3 ). When extended over other regimes, this finding also implies that even occupying similar dynamic volumes the slow-twitched fibres and fast-twitched fibres interact by different ways producing distinct force output, as discussed in Equation (24). In the maximum-velocity regime $r=3$, the secondary function coincides with the primary function for the case of strut muscle (see items 5 and 6), whereas the brake muscle may efficiently work as motor. New secondary activities are exposed by the motor, which also may function as "the fastest motor", determined by $\eta_{6}=(6 / 7) /(1 / 7)=6$, and by the cardiac muscle, which fast-velocity action may suggest, say, a "sharp-heart" accommodation associated with $\eta_{0}=0$, formally opposed to that of the strut $\left(\eta_{3}=\infty\right)$.

\subsection{Direct Observations of Muscle Specialization}

"If a muscle is specialized for a particular mechanical role how this is reflected in it architecture?" [45]. The proposed solution to stated problem is demonstrated below by the comparative analysis between the muscle architecture observed by allometric exponents and that predicted by the adaptation to a particular mechanical function.

\subsubsection{Isolated Muscles in Hindlimb of Mammals and Birds}

In Table 5 we analyze the morphometric data on the allometric exponents derived from the mean cross-sectional area $A_{0 m}^{(\text {exp })}$ and length $L_{0 m}^{(\exp )}$ of four skeletal muscles in the mammalian hindlimb for 35 quadrupedal species of body-mass domain exceeding four orders in magnitude.

First, let us verify the cylinder-shape similarity of skeletal muscles described by Equation (9). The muscle mass index $\alpha_{0 m}$ estimated in Equation (10) via experimental data $a_{0 m}^{(\exp )}$ and $l_{0 m}^{(\exp )}$ is compared in Table 5 with the measured indexes $\alpha_{0 m}^{(\exp )}$.

In Figure 2 and Figure 3, the method of determination of the primary mechanical function is illustrated: the adapted muscle structure is indicated by the appropriate theoretical point located most closely to the datapoint.

The found reliable estimates $\alpha_{0 m}^{(e s t)}$ were used then in the muscle-function analysis in Figure 2 and Figure 3. The established small indices $\alpha_{0 m}$ generally validate the muscle biomechanics by proving a high-precision observation of locomotory muscle patterns via muscle morphometry and functional physiology. This implies that the effect of biomechanical adaptation of muscle design to active elastic forces predominates over effects of biological adaptation assigned to small $\alpha_{0 m}^{(\exp )}$.

Secondly, the analysis in Figure 2 and Figure 3 indicates strong correlations between the morphometrically characterized structure of skeletal muscle and one of the primary locomotor functions described in Table 2. The 
primary functions indicated in Table 5 are found with a high degree of certainty. Indeed, as illustrated in Figure 2, the deviations of distances measured along the dashed line, corresponding to a given muscle, between the datapoint and distant challengers for the primary function, from the smallest distance indicating the primary candidate, always exceed the experimental uncertainty.

Thirdly, the found muscle mechanical specifications do not conflict with the physiological categorization established for joint extensors and flexors, which muscle structures are shown to be adapted to the brake and motor functions via activation of eccentric and concentric elastic forces. The found structure parameter $\eta_{\text {plant }} \approx 18$ indicates the foot support activity for plantaris as the primary function (Table 5) that is in accord with in vivo workloop presented in the inset c in Figure 1. As shown in Table 3, the struts are most conservative muscles

Table 5. The analysis of the allometric data by Pollock and Shadwick [26] provided on the basis of Equation (10) and Table 2. The shown statistical error is approximated by the symmetrized $95 \%$ confidence interval. The methodology of the analysis is illustrated in Figure 2. The primary functions found in Figure 2 and Figure 3 are described following Table 2, with $\alpha_{m}=\alpha_{0 m}^{(\text {est })}$. The overall muscle group $(g=1)$ is determined as the standard mean over all muscles. ${ }^{*}$ DDF includes individual flexor hallucis and flexor digitorum longus; SDF means superficial digital flexor.

\begin{tabular}{|c|c|c|c|c|c|c|c|c|}
\hline $\begin{array}{c}\text { Individual } \\
\text { mammalian } \\
\text { muscle }\end{array}$ & $a_{0 m}^{(\exp )}$ & $l_{0 m}^{(\exp )}$ & $\alpha_{0 m}^{(\exp )}$ & $\eta_{0 m}$ & $\alpha_{0 m}^{(e s t)}$ & $a_{m}$ & $l_{m}$ & Primary funct. \\
\hline $\begin{array}{l}\text { Gastrocnemius } \\
\text { (and soleus) }\end{array}$ & $0.77 \pm 0.02$ & $0.21 \pm 0.02$ & -0.03 & 3.7 & -0.02 & 0.78 & 0.20 & motor, $\quad m=1$ \\
\hline $\begin{array}{l}\text { Deep digital } \\
\text { flexor (DDF) }\end{array}$ & $0.85 \pm 0.03$ & $0.18 \pm 0.02$ & 0.03 & 4.7 & 0.03 & 0.82 & 0.21 & motor, $m=1$ \\
\hline $\begin{array}{l}\text { Common digital } \\
\text { extensor (CDE) }\end{array}$ & $0.69 \pm 0.04$ & $0.24 \pm 0.02$ & -0.07 & 2.9 & -0.07 & 0.70 & 0.23 & brake, $m=2$ \\
\hline Plantaris (SDF) & $0.91 \pm 0.04$ & $0.05 \pm 0.04$ & -0.03 & 18 & -0.04 & 0.96 & 0.00 & strut, $m=3$ \\
\hline $\begin{array}{l}\text { Ankle-joint } \\
\text { muscle group }\end{array}$ & $0.81 \pm 0.03$ & $0.17 \pm 0.03$ & -0.03 & 4.8 & -0.03 & 0.78 & 0.19 & motor, $g=1$ \\
\hline
\end{tabular}

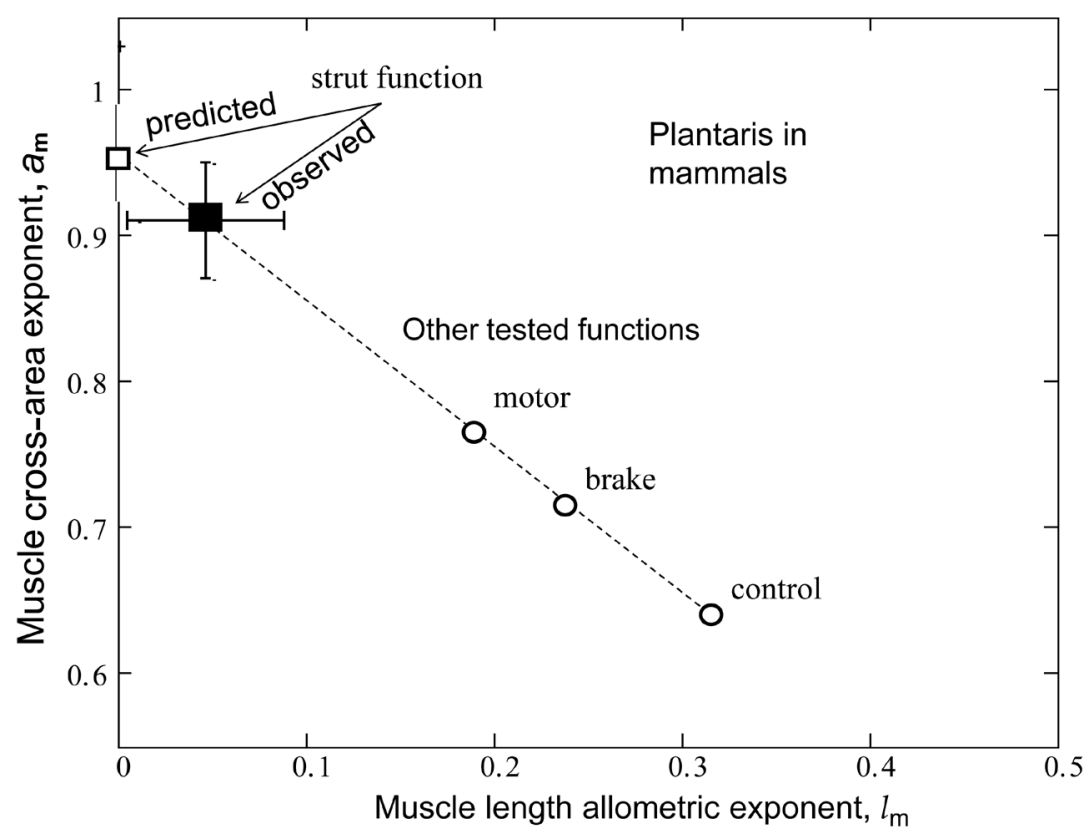

Figure 2. The indirect observation of the primary activity of mammalian plantaris. The solid symbol is the datapoint [26] presented in Table 5 and the bars indicate experimental error. The open symbols are theoretical estimates for stable dynamic structures established for the motor, brake, strut, or control functions described in Table 2, with $\alpha_{m}=\alpha_{0 m}^{(\text {est })}$ taken from Table 5 . 


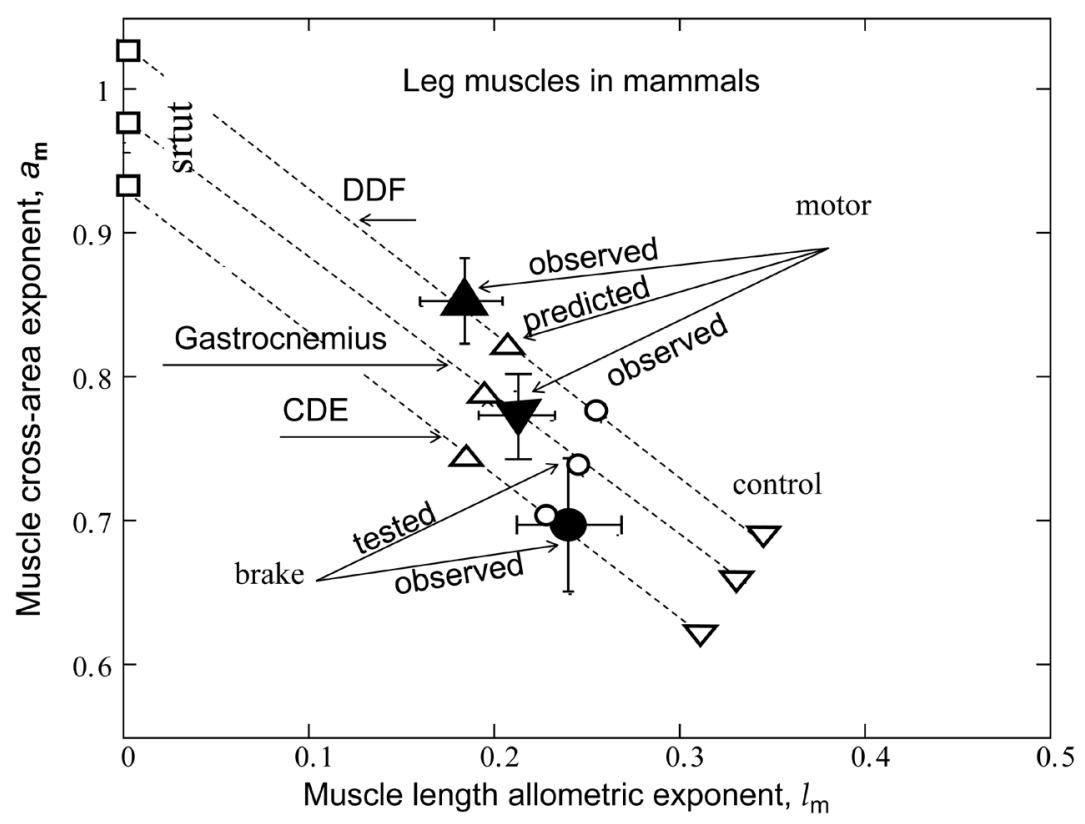

Figure 3. The observation of the primary mechanical function in some isolated individual muscles in mammals. The analysis and notations correspond to those in Figure 2. The experimental (and theoretical) data for gastrocnemius, DDF (deep digital flexor), and CDE (common digital extensor) are shown, respectively, by the closed (and open) inverted triangles, regular triangles, and circles. All the data are taken from Table 5 .

no changing their support function in non-linear regimes. In contrast, the gastrocnemius in mammals manifests their motor, strut, and brake functions in, respectively, uphill, level, and incline running of animals. Through the motor adapted structure with $\eta_{\text {gast }} \approx \eta_{1}=4$, the analysis in Figure 3 establishes the motor activity for gastrocnemius as the primary function naturally selected for the significant mechanical task of uphill running exploring the bilinear muscle dynamics. The effective trilinear gastrocnemius-displacement dynamics is most close to the brake-like activity $\left(\eta_{6}=6\right)$, attributed to the secondary function of the motor experimentally observed in gastrocnemius of incline running turkey [31] and hopping tammar wallabies [28].

In Figure 4, the overall muscle peak stress data measured in limb muscles of animals in strenuous activity, reviewed by Biewener [29], are re-examined and re-analyzed accounting for the primary functions of hindlimb muscles established in Table 5.

The uphill-motor specialization of gastrocnemius is independently supported by the compressive-stress analysis made in Figure 4 for fast running, jumping, and hopping mammals. The stress scaling exponents $\left(s_{m}\right)$ predicted for the motor $\left(s_{1}=1 / 5\right)$, strut $\left(s_{3}=0\right)$, and control $\left(s_{4}=0\right)$ functions are shown to be distinguishable in work-specific mammalian muscles described in Table 2. Hence, although the overall-function data by Biewener [29] indeed expose almost weight-independent muscle stress, earlier postulated by McMahon in Equation (5) and only in part justified here by the slow-fibre muscles (Table 1) and strut muscles (Table 2), the analyses in Figure 4 demonstrates how the function-specific muscle stress may serve as a new tool for the direct observation of muscle specialization generally ignored in all previous overall-function analyses.

I have also investigated an interesting question: whether the primary function established for a certain leg muscle in mammals specialized to fast running coincides with that for the same muscle in birds? The pioneering data on individual leg muscles in 8 running birds, ranging in size from $0.1 \mathrm{~kg}$ quail to $40 \mathrm{~kg}$ ostrich, are analyzed in Table 6 and Figure 5.

In running and non-running birds (Figure 5), the gastrocnemius is employed as the brake and spring, in contrast to the motor function in mammals (Table 5). This is in accord with Bennett [27], who noted that "the full force-generated capacity of gastrocnemius is only used occasionally, such as during take-off, when a bird attempts to throw itself into the air". This explains our indirect observation: the primary function of the gastrocnemius in running specialists is attributed to the foot flexor in mammals and ankle extensor in birds (Table 6). 
In non-running birds, the legs are designed to control the ground locomotion (Figure 5), whereas their wings may share motor and brake functions (Table 3), in accord with that reviewed by Dickinson et al. [6].



Figure 4. The qualitative study of the in vivo data on the peak stress in individual leg muscles of animals in strenuous activity. The symbols employed above in Figure 2 and Figure 3 are extended by the open circles (triceps) for the data on peak muscle stress taken from Table 1 in [29], with the exclusion of the slow-mode data on cantering goat and trotting cat. The data [46] on the activated isometric stress in isolated white rabbit tibialis are added. The dashed line shows the brake-functional stress indicated by the stress scaling exponent $s=1 / 4$. The solid lines are drawn by $115 \cdot M^{1 / 5}$, for the motor function, and by $215 \mathrm{kPa}$, for the strut and spring functions. All coefficients are adjusted by eye.

Table 6. The analysis of the allometric data by Maloiy et al. [47]. The shown large error is due to relatively wide confidence limits. The mean exponents $l_{0 m}^{(e s t)}$ are estimated via Equation (10). The overall muscle group is determined as the standard mean over all muscles. The indicated primary functions and active elastic forces are described by the evaluated dynamic-structure exponents $a_{2 m}$ and $l_{2 m}$ found as most close to the experimental resting-volume data on $a_{0 m}^{\text {(exp) }}$ and $l_{0 m}^{\text {(exp) }}$ and therefore assigned to regime $r=2$ (Table 2).

\begin{tabular}{|c|c|c|c|c|c|c|c|}
\hline Running birds & $a_{0 m}^{(\exp )}$ & $\alpha_{0 m}^{(\exp )}$ & $l_{0 m}^{(e s t)}$ & $a_{0 m}^{(\text {exp) }} / l_{0 m}^{(\text {est })}$ & $a_{2 m}$ & $l_{2 m}$ & $\begin{array}{l}\text { Primary function } \\
\text { (force) }\end{array}$ \\
\hline Gastrocnemius & $0.81 \pm 0.14$ & 0.14 & 0.33 & 2.5 & 0.85 & 0.29 & brake (eccentric) \\
\hline $\begin{array}{l}\text { Digital flexors } \\
\text { (DF) }\end{array}$ & $0.76 \pm 0.22$ & -0.0 .3 & 0.21 & 3.6 & 0.78 & 0.19 & motor (concentric) \\
\hline Femorotibialis & $0.80 \pm 0.12$ & -0.02 & 0.18 & 4.4 & 0.78 & 0.20 & motor (concentric) \\
\hline Overall group & $0.79 \pm 0.16$ & 0.03 & 0.24 & 3.3 & 0.77 & 0.26 & brake (eccentric) \\
\hline
\end{tabular}




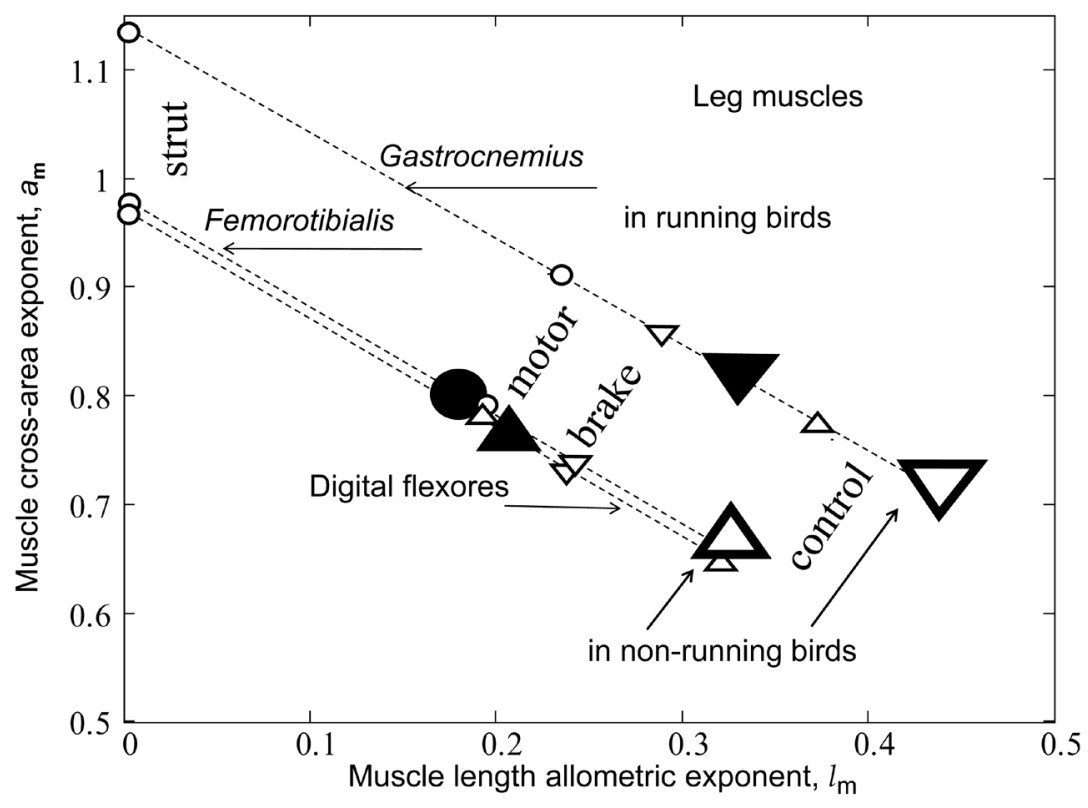

Figure 5. The analysis of the primary mechanical functions for leg muscles in running and non-running birds. The measured (and estimated) data taken from Table 6 (and Table 2) for gastrocnemius, femorotibialis, and digital flexors are shown by the closed (and open) inverted triangles, circles, and regular triangles, respectively. The semi-open triangles are the data by Bennett [27] for non-running birds.

\subsubsection{Micro-Macro Scale Correspondence}

There are many striking examples when skeletal muscles expose adaptation to a specific function, e.g. [3] [48]. The striated muscles anatomically suited to concentric or eccentric work [2] are structurally distinct having, respectively, long thin cells or short wide cells [5]. This observation suggests the microscopic level of muscle-cell adaptation introduced here by

$$
A_{\text {cell }}^{(\text {conc) }}>A_{\text {cell }}^{(\text {ecent })} \text { and } L_{\text {cell }}^{(\text {ecent })}>L_{\text {cell }}^{(\text {conc })}
$$

for the cellular cross-sectional area $A_{\text {cell }}$ and length $L_{\text {cell }}$. Adopting these function specific trends, one may expect to observe the cell-structure parameters $\eta_{\text {cell }}=4$ and 3 for sarcomeres accommodated to efficient shortening or stretching of muscle as a whole.

A general question arises whether allometric coefficients of proportionality omitted above in all structurefunction power-law (scaling) relations are also attributed to active elastic strains accompanying maximum force production? Or, alternatively, other microscopically justified mechanisms, c.f. [49], or additional parameters (such as pinnate angle) may result in different general macroscopic consequences? Given the highly conservative nature of contractive units of skeletal muscles and their well pronounced organization [29], the specific-function trends of the muscle cross-sectional area

$$
A_{\text {strut }}^{\text {(isom) }}>A_{\text {motor }}^{\text {(conc) }}>A_{\text {brake }}^{\text {(eccen) }}>A_{\text {contr }}^{\text {(sprin) }}
$$

and muscle-fibre length

$$
L_{\text {contr }}^{(\text {sprin })}>L_{\text {brake }}^{(\text {eccen })}>L_{\text {motor }}^{(\text {conc) }}>L_{\text {strut }}^{\text {(isom) }}
$$

are generally expected from Table 2 . The suggested trends become observable via the primary functions established in Table 5 for gastrocnemius $(m=1)$, DDF $(m=1)$, CDE $(m=2)$, and plantaris $(m=3)$, when the regression data [26] on passive-muscle structure $\left[A_{0 m}^{(\exp )}(M), L_{0 m}^{(\exp )}(M)\right]$ are taken additionally into consideration: $A_{\text {plant }}^{(\exp )}>A_{\text {gast }}^{(\exp )} \gtrsim A_{D D F}^{(\exp )}>A_{C D E}^{(\exp )}$ and $L_{C D E}^{(\exp )}>L_{\text {gast }}^{(\exp )} \gtrsim L_{D D F}^{(\exp )}>L_{\text {plant }}^{(\exp )}$, starting with $M>1 \mathrm{~kg}$.

Similarly, the trend for active stiffness

$$
K_{\text {strut }}^{(\max )}>K_{\text {motor }}^{(\max )}>K_{\text {brake }}^{(\max )} \text { and, generally, } K_{\text {fast }}^{(\max )}>K_{\text {slow }}^{(\max )}
$$


straightforwardly follows from Table 2. Given that the optimum velocity for fast fibres $V_{1 m} \propto L_{m}^{1 / 2}$ (Table 1), Equation (31) provides

$$
V_{\text {brake }}^{(\text {opt })}>V_{\text {motor }}^{(o p t)}>V_{\text {strut }}^{(\text {opt })}
$$

Moreover, a crude estimate for the cost energy

$$
C U_{\text {motor }}^{(\max )}>C U_{\text {strut }}^{(\max )}>C U_{\text {brake }}^{(\max )}
$$

follows from $C U_{\text {fast }}^{(\max )} \propto M_{m}$ in Equation (22) and the experimental data by Pollock and Shadwick [26], $M_{1}^{(\exp )}>M_{3}^{(\exp )}>M_{2}^{(\exp )}$, considered at the same body mass $M$. The finding in Equation (34) is in accord with the experimental observation [49]: muscles contracting nearly isometrically (strut function) generate force more economically than muscles involved in concentric work (via motor function).

\subsubsection{Muscle Dynamics of Mammalian Legs and Dragonfly Wings}

Given that mammalian leg extensors are active mostly during lengthening [2], the brake primary function ( $m=2$ in Table 2) could be assigned to leg muscles specified by effective length $L_{\text {leg }} \propto M^{1 / 4} \quad\left(\alpha_{\text {leg }}=0\right.$ is adopted). In accord with Hill's second velocity-inverse-length constraint, underlaid by the proper frequency $T_{3 s \text { sow }}^{-1} \propto L_{m}^{-2}$ in the maximum-velocity regime (Table 4), the theory predicts $V_{\text {leg }}^{(\max )} \propto L_{\text {leg }}^{-1} \propto M^{-1 / 4}$ and $1 / T_{\text {leg }}^{(\max )} \propto L_{\text {leg }}^{-2} \propto M^{-1 / 16}$, for steady locomotory modes required slow-fibre muscles [40]. Similarly, for the wing-motor muscles in flying birds ( $m=1$ in Table 2) one should expect $V_{\text {wing }}^{(\max } \propto L_{\text {wing }}^{-1} \propto M^{-1 / 5}$, for contraction velocity, and $1 / T_{\text {wing }}^{(\max )} \propto M^{-1 / 25}$, for the frequency or, alternatively, $1 / T_{\text {wing }}^{(\text {opt }} \propto M^{-1 / 5}$, in the optimum-velocity regime (see Table 4). Hence, analytically revealed Hill's constraint becomes observable via the empirical regression data by Medler [48]: on the maximum-amplitude contraction velocities for the locomotor muscles in leg of terrestrial animals, $V_{\text {leg }}^{(\text {exp }} \propto M^{-0.25}$, and that for wings in flying birds, bats, and insects, $V_{\text {wing }}^{(\text {exp }} \propto M^{-0.20}$. Moreover, the proposed theory sheds light on other empirical data on the maximum velocity in fast running animals $V_{\max }^{(\exp )} \propto M^{-0.125}$ [48] [49] successfully explained by $V_{\max }^{(p r e)} \propto M^{-1 / 8}$ predicted by McMahon's elastic similarity model [19]. As follows from Table 4, both McMahon's result and the data now are specified by the maximum-velocity regime, with $V_{3 \text { fast }}^{(\max } \propto L_{\text {leg }}^{-1 / 2}$, required fast-fibre leg muscles during non-steady transient modes [40] [43]. Likewise, the regression analysis made in Figure 6 for the motor-flight muscle of resting length scaling as $L_{0 m} \propto M_{m}^{1 / 5}$, indicates that the data on the wingbeat frequency $1 / T_{\text {wing }}^{(\text {exp }} \propto M_{m}^{-0.20}$
[50] were obtained in dragonflies, flying in steady regimes at optimum-amplitude velocities.

In the same optimum-velocity regime (Table 1), the maximum-amplitude static muscle force $F_{\text {stat }}^{(\text {exp })} \cong \Delta F_{1 m}^{\text {(slow) }} \propto M_{m}^{2 / 3}$ and net lever-system force $F_{\text {ind }}^{(\text {(exp })} \cong \Delta F_{1 m}^{(\text {fast })} \propto M_{m}$ reported by Schilder and Marden [50] may be associated with the slow and fast activated fibres in the basalar muscles tuned elastically to the linear regime through the muscle dynamic volume provided by PCSA $A_{1 m}^{(d y n)} \propto M_{m}^{2 / 3}$ and length $L_{1 m}^{(d y n)} \propto M_{m}^{1 / 3}$. The observed dynamic force output $F_{d y n}^{(\exp )} \propto M_{m}^{0.83}$ can be therefore suggested as the mixed-fibre force $F_{d y n}^{(\text {pred })} \cong \Delta F_{1 m}^{(\text {mix })} \propto M_{m}^{5 / 6} \quad$ (Table 1), i.e. as a compromise of the slow force output $F_{m}^{\text {(slow) }}$ and fast force output $F_{m}^{(\text {fast })}$. These estimates challenge further analysis of the basalar in vitro force-length loops (workloops) studied in $[50]$.

\section{Conclusions}

A theoretical framework for the mechanical characterization of the three activated states of the striated muscle introduced via force-length cycling in terms of the three well distinguished dynamic transient regimes is proposed. The explicit analytical description of muscle locomotor functions and related mechanical characteristics is provided on the basis of two concepts: 1) the preservation of dynamic muscle volume associated with self-preservation of the spindle-type shape in skeletal muscles and egg-type shape in cardiac muscles 2) the mechanical similarity between action (loading) and reaction (elastic) forces takes place in biomechanically equivalent states. Exploring a full set of a few numbers of patterns known for elastic forces from the continuum mechanics, the macroscopic study of the force production and its functional-structural accommodation in the loaded muscle organs provides the following major outcomes.

1) It is demonstrated how the generic principle of mechanical (force and velocity) similarity provides the dynamic (frequency-velocity) constraints for the muscle contractions, first observed by Hill in hovering birds and then revealed in locomotor muscles of running animals and flying birds, bats, and insects. 


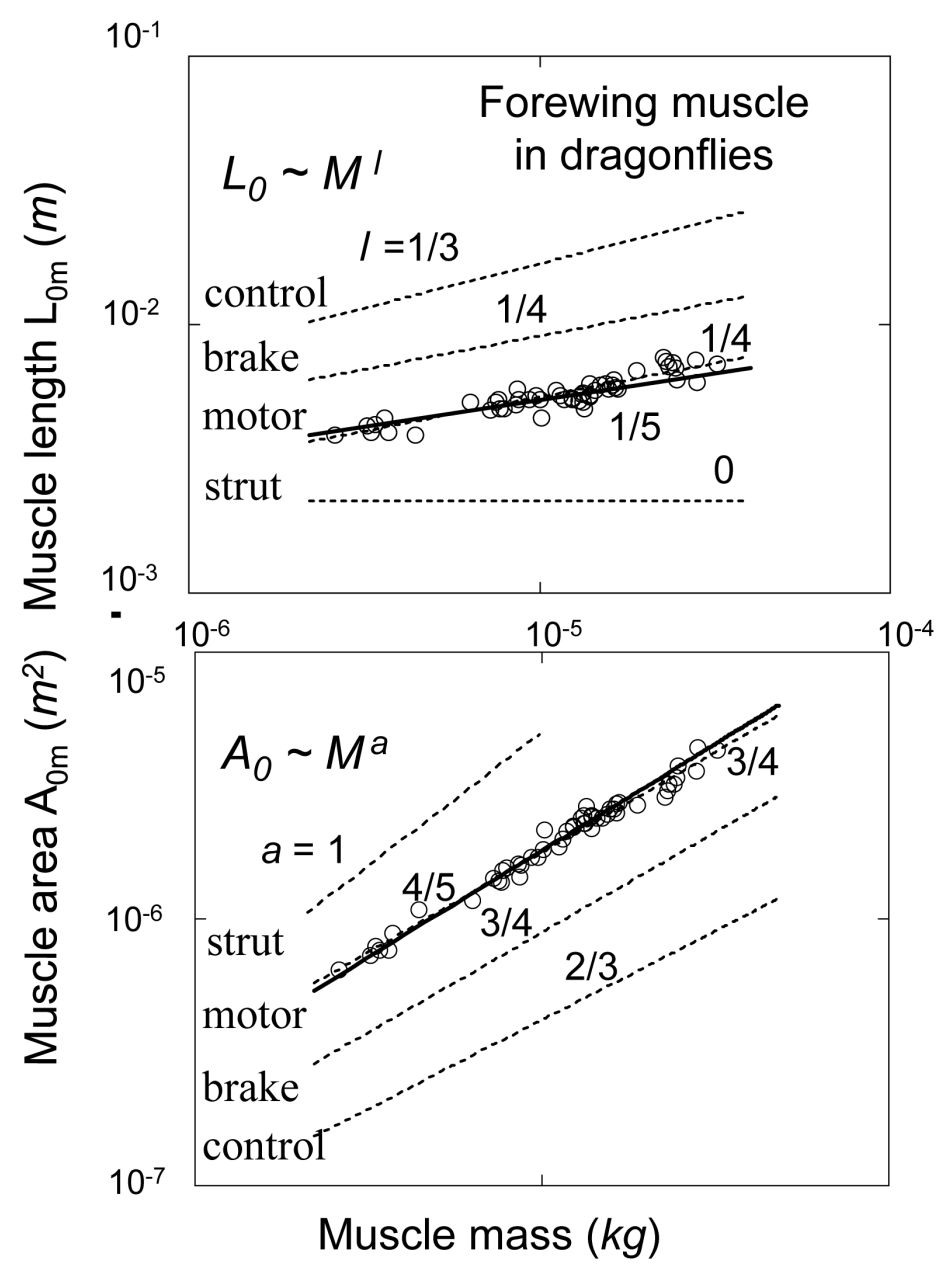

Figure 6. Scaling of the basalar structure to muscle mass in male dragonflies (Odonata and Anisoptera, listed in Figure 5 in [50]). The datapoints for muscle length $L_{0 m}^{(\exp )}$ is a courtesy by the authors. The estimated muscle cross-sectional area $A_{0 m}^{(e s t)}$ is obtained on the basis of Equation (1) taken with $\rho_{m}^{(\exp )}=1060 \mathrm{~kg} / \mathrm{m}^{3}$ [51]. The solid lines are $L_{\text {motor }}=0.052 \cdot M_{m}^{1 / 5}$ and $A_{\text {motor }}=0.018 \cdot M_{m}^{4 / 5}$. The dashed lines indicated by other scaling exponents are drawn according to muscle specialization shown in Table 2. All pre-exponential coefficients are adjusted by eye.

2) It is shown how the standard relations from classical mechanics of solids may successfully work in soft tissue mechanics. The study is grounded by the active-force muscle stiffness well distinguished in muscle workloops near the maximum-amplitude exerted forces. At a fixed muscle shape, the muscle active stiffness, underlaid by sarcomere stiffness, is shown to be driven by the elastic moduli, which encompass all contractive elements arranged here as the elastic continuum medium.

3) The provided analysis of direct empirical observations of the maximal forces exerted by muscles in legs of mammals and birds and the scaling analysis of the resting muscle structures corroborates the major hypothesis of the study: the force output amplitude in high powering fast and slow individual muscles is linear with, respectively, muscle volume and cross-sectional area, regardless of their muscle's specialization specified by primary functions.

4) The study reveals that the mechanical similarity applied to locomotor skeletal muscles specialized in spring, brake, and motor activities resembles McMahon’s "geometric", "elastic", and "static” stress similarities. It is not 
striking since the employed here scaling patterns for reaction elastic forces are well established in continuum mechanics of solids regardless of the nature of origin of other mechanical forces, e.g. external "passive" gravitational-field forces or internal "active" muscular-field forces. As the result, several observations of empirical data matching McMahon’s predictions, e.g. [48] [49], are illuminated in terms of distinct muscle dynamic regimes.

5) The macroscopic structures of locomotor skeletal muscles revealed by the muscle allometry are found to be well adapted to the dynamic state with generation of maximum force at moderate velocity contractions. The relevant bilinear-displacement muscle dynamics, involving both fast-twitch and slow-twitch powering muscle fibres sheds light on the origin of allometric power laws and explains muscle specialization. The adapted structures examined via the available empirical data indicate that the leg muscles are brakes in mammals and springs in non-running birds, whereas the wing muscles are motor-brake engines in flying species. A new pump function suggested for the cardiac muscles needs further experimental tests.

6) The provided study of the muscle specialization in mammalian hindlimb indicates that the force production function is a dominated factor in the accommodation of muscle structure. When the mechanical scaling exponents are compared with the relatively small muscle-mass index, this finding likely indicates the predominating role in mass distribution due to mechanical adaptation effects over that due to biological adaptive mechanisms. As the result, a new tool for the investigation of indirect effects of the biomechanical adaptation of individual locomotor muscles is suggested through the regression analysis of in vivo muscle stresses in synergists scaled across different-sized animals.

7) The assumption on that the muscle tuning muscle ability of animals can be modeled by active elastic forces via non-linear muscle elastic moduli is validated through the observation of theoretical predictions made in muscle dynamics for legs and wings in running and flying specialists. Predictions are also provided for the experimental testing of the primary and secondary functions realized by the tuning the cycling muscle to the proper natural frequency.

8) The conservative character of architecture and related mechanical characteristics of striated muscles suggests general trends following from the mechanical and shape constraints. The trends dictated by the primary functions explain, in particular, why the muscles having larger fibre and sarcomere lengths and suited to efficient eccentric work, tend toward higher optimum contraction velocities, but show lower maximum stiffness and mechanical energy cost.

9) As an intriguing outcome of the analysis of maximal contraction muscle velocities and frequencies, the maximum-speed steady locomotion is revealed to be controlled by non-linear elasticity of slow-fibre muscles generating moderated force. This finding deserves further evaluation in finite muscle element analysis when studying top speeds of living and extant animals.

\section{Acknowledgements}

The author thanks Robert McN. Alexander, Andrew A. Biewener and James H. Marden for careful reading of the first versions of this study and helpful critical comments. Rudolf J. Schilder is appreciated for the datapoints on dragonflies. This work was in part sponsored by the national agency CNPq.

\section{References}

[1] Hill, A.V. (1950) The Dimensions of Animals and Their Muscular Dynamics. Science Progress, 38, 209-230.

[2] Lindstedt, S.L., Trude, E.R., Paul, K. and Paul, C.L. (2002) Do Muscle Function as Adaptable Locomotor Springs? Journal of Experimental Biology, 205, 2221-2216.

[3] Lichtwark, G.A. and Wilson, A.M. (2005) Effects of Series Elasticity and Activation Conditions on Muscle Power Output and Efficiency. Journal of Experimental Biology, 208, 2845-2853. http://dx.doi.org/10.1242/jeb.01710

[4] Squire, J.M. (1997) Architecture and Function in the Muscle Sarcomere. Current Opinion in Structural Biology, 7, 247-257. http://dx.doi.org/10.1016/S0959-440X(97)80033-4

[5] Russell, B., Motlagh, D. and Ashley, W.W. (2000) Form Follows Function: How Muscle Shape Is Regulated by Work. Journal of Applied Physiology, 88, 1127-1132. http://www.jappl.org/content/88/3/1127.full

[6] Dickinson, M.H., Farley, C.T., Full, J.R., Koehl, M.A.R., Kram, R. and Lehman, S. (2000) How Animals Move: An Integrative View. Science, 288, 100-106. http://dx.doi.org/10.1126/science.288.5463.100

[7] Rome, L.C. (2006) Design and Function of Superfast Muscles: New Insights into the Physiology of Skeletal Muscle. Annual Review of Physiology, 68, 193-221. http://dx.doi.org/10.1146/annurev.physiol.68.040104.105418 
[8] Medler, S. and Hulme, K. (2009) Frequency-Dependent Power Output and Skeletal Muscle Design. Comparative Biochemistry and Physiology Part A: Molecular \& Integrative Physiology, 152, 407-417. http://dx.doi.org/10.1016/j.cbpa.2008.11.021

[9] Jontes, J.D. (1995) Theories of Muscle Contraction. Journal of Structural Biology, 115, 119-143. http://dx.doi.org/10.1006/jsbi.1995.1037

[10] Cole van den, G.K., Bogert, A.J., Herzog, W. and Gerritsen, K.G.M. (1996) Modelling of Force Production in Skeletal Muscle Undergoing Stretch. Journal of Biomechanics, 29, 1091-1104. http://www.ingentaconnect.com/content/els/00219290/1996/00000029/00000008/art00005

[11] Alexander, R.McN. (1977) Allometry of the Limbs of Antelopes (Bovidae). Journal of Zoology (London), 183, 125146. http://dx.doi.org/10.1111/j.1469-7998.1977.tb04177.x

[12] Forcinito, M., Epstein, M. and Herzog, W. (1998) Can a Rheological Muscle Model Predict Force Depression/Enhancement? Journal of Biomechanics, 31, 1093-1099. http://dx.doi.org/10.1016/S0021-9290(98)00132-8

[13] Kokshenev, V.B. (2008) A Force-Similarity Model of the Activated Muscle Is Able to Predict Primary Locomotor Functions. Journal of Biomechanics, 41, 912-915. http://dx.doi.org/10.1016/j.jbiomech.2007.11.005

[14] Jenkyn, T.R., Koopman, B., Huijing, P., Lieber, R.L. and Kaufman, K.R. (2002) Finite Element Model of Intramuscular Pressure during Isometric Contraction of Skeletal Muscle. Physics in Medicine and Biology, 47, 4043-4061. http://dx.doi.org/10.1088/0031-9155/47/22/309

[15] Marra, S.P., Ramesh, K.T. and Douglas, A.S. (2003) Characterization and Modeling of Compliant Active Materials. Journal of the Mechanics and Physics of Solids, 51, 1723-1743. http://dx.doi.org/10.1016/S0022-5096(03)00055-3

[16] Skatulla, S., Arockiarajan, A. and Sansour, C. (2009) A Nonlinear Generalized Continuum Approach for Electro-Elasticity Including Scale Effects. Journal of Mechanics and Physics of Solids, 57, 137-160. http://dx.doi.org/10.1016/j.jmps.2008.09.014

[17] Dumont, E.R., Grosse, I.R. and Slater, G.J. (2009) Requirements for Comparing the Performance of Finite Element Models of Biological Structures. Journal of Theoretical Biology, 256, 96-103. http://dx.doi.org/10.1016/j.jtbi.2008.08.017

[18] McMahon, T.A. (1973) Size and Shape in Biology. Science, 179, 1201-1204. http://dx.doi.org/10.1126/science.179.4079.1201

[19] McMahon, T.A. (1975) Using Body Size to Understand the Structural Design of Animals: Quadrupedal Locomotion. Journal of Applied Physiology, 39, 619-627. http://www.deepdyve.com/lp/the-american-physiological-society/using-body-size-to-understand-the-structural-designof-animals-ac2OYKkNgX

[20] Kokshenev, V.B. (2003) Observation of Mammalian Similarity through Allometric Scaling Laws. Physica A, 322, 491-505. http://dx.doi.org/10.1016/S0378-4371(02)01923-4

[21] Kokshenev, V.B. and Christiansen, P. (2010) Salient Features in Locomotion of Proboscideans Revealed via the Differential Scaling of Limb Long Bones. Biological Journal of Linnean Society, 100, 16-29. http://dx.doi.org/10.1111/j.1095-8312.2010.01415.x

[22] Kokshenev, V.B. and Christiansen, P. (2011) Evolution of Locomotor Trends in Extinct Terrestrial Giants Affected by Body Mass. In: Klika, V., Ed., Theoretical Biomechanics, InTech, Croatia, 49-74. http://cdn.intechweb.org/pdfs/22186.pdf

[23] Bejan, A. and Marden, J.H. (2006) Unifying Constructal Theory for Scale Effects in Running, Swimming and Flying. Journal of Experimental Biology, 209, 238-248. http://dx.doi.org/10.1242/jeb.01974

[24] Kokshenev, V.B. (2011) Physical Insights into Dynamic Similarity in Animal Locomotion. I. Theoretical Principles and Concepts; II. Observation of Continuous Similarity States. In: Klika, V., Ed., Theoretical Biomechanics, InTech, Croatia, 267-302. http://www.intechopen.com/download/get/type/pdfs/id/22195

[25] Alexander, R.McN. (1997) Optimum Muscle Design for Oscillatory Movements. Journal of Theoretical Biology, 184, 253-259. http://dx.doi.org/10.1006/jtbi.1996.0271

[26] Pollock, C.M. and Shadwick, R.E. (1994) Allometry of Muscle, Tendon, and Elastic Energy-Storage Capacity in Mammals. American Journal of Physiology, 266, R1022-R1031.

[27] Bennett, M.B. (1996) Allometry of the Leg Muscles in Birds. Journal of Zoology (London), 238, 435-443. http://dx.doi.org/10.1111/j.1469-7998.1996.tb05404.x

[28] Biewener, A.A., Konieczynski, D.D. and Baudinette, R.V. (1998) In Vivo Muscle Force-Length Behavior during Steady-Speed Hopping in Tammar Wallabies. Journal of Experimental Biology, 201, 1681-1694.

[29] Biewener, A.A. (2005) Biomechanical Consequences of Scaling. Journal of Experimental Biology, 208, $1665-1676$. http://dx.doi.org/10.1242/jeb.01520 
[30] Kokshenev, V.B. (2007) New Insights into Long-Bone Biomechanics: Are Limb Safety Factors Invariable across Mammalian Species? Journal of Biomechanics, 40, 2911-2918. http://dx.doi.org/10.1016/j.jbiomech.2007.03.007

[31] Roberts, T.J., Marsh, R.I., Weyand, P.G. and Taylor, C.R. (1997) Muscular Force in Running Turkey: The Economy of Minimizing Work. Science, 275, 1113-1115. http://dx.doi.org/10.1126/science.275.5303.1113

[32] Rome, L.C. (1997) Testing a Muscle’s Design. American Scientist, 85, 356-363. http://www.jstor.org/discover/10.2307/27856813?uid=3737664\&uid=2\&uid=4\&sid=21103234444773

[33] Josephson, R.K. (1999) Dissecting Muscle Power Output. Journal of Experimental Biology, 202, 3369-3375.

[34] Ahlborn, B.K., Blake, R.W. and Megill, W.M. (2006) Frequency Tuning in Animal Locomotion. Zoology, 109, 43-53. http://dx.doi.org/10.1016/j.zool.2005.11.001

[35] Forcinito, M., Epstein, M. and Herzog, W. (1997) Theoretical Considerations on Myofibril Stiffness. Biophysical Journal, 72, 1278-1286. http://dx.doi.org/10.1016/S0006-3495(97)78774-5

[36] Robinson, J.M., Wang, Y., Kerrick, W.G.L., Kawai, R. and Cheung, H.C. (2002) Activation of Striated Muscle: NearestNeighbor Regulatory-Unit and Cross-Bridge Influence on Myofilament Kinetics. Journal of Molecular Biology, 322, 1065-1088. http://dx.doi.org/10.1016/S0022-2836(02)00855-0

[37] Landau, L.D. and Lifshitz, E.M. (1989) Theory of Elasticity. Pergamon Press, London.

[38] Kokshenev, V.B. (2010) Key Principle of the Efficient Running, Swimming, and Flying. Europhysics Letters, 90, 48005-p1-p5. http://dx.doi.org/10.1209/0295-5075/90/48005

[39] Kent, G.C. (1987) Comparative Anatomy of the Vertebrates. Wm. C. Brown Publishers, Dubuque.

[40] Rome, L.C., Runke, R.P., Alexander, R.M., Lutz, G., Aldridge, H., Scott, F. and Freadman, M. (1988) Why Animals Have Different Fibre Types. Nature, 335, 824-827. http://dx.doi.org/10.1038/335824a0

[41] Farley, C.T., Glasheen, J. and McMahon, T.A. (1993) Running Springs: Speed and Animal Size. Journal of Experimental Biology, 185, 71-86.

[42] Bejan, A. and Marden, J.H. (2006) Unifying Constructal Theory for Scale Effects in Running, Swimming and Flying. Journal of Experimental Biology, 209, 238-248.

[43] Rome, L.C., Sosnicki, A.A. and Goble, D.O. (1990) Maximum Velocity of Shortening of Three Fibre Types from Horse Soleus Muscle: Implications for Scaling with Body Size. Journal of Physiology, 431, 173-185.

[44] Marden, J.H. and Allen, L.R. (2002) Molecules, Muscles, and Machines: Universal Performance Characteristics of Motors. Proceedings of the National Academy of Sciences of the United States of America, 99, 4161-4166. http://dx.doi.org/10.1073/pnas.022052899

[45] Daley, M.A. and Biewener, A.A. (2003) Muscle Force-Length Dynamics during Level versus Incline Locomotion: A Comparison of in Vivo Performance of Two Guinea Fowl Ankle Extensors. Journal of Experimental Biology, 206, 2941-2958. http://dx.doi.org/10.1242/jeb.00503

[46] Davis, J., Kaufman, K.R. and Lieber, R.L. (2003) Correlation between Active and Passive Isometric Stress and Intramuscular Pressure in the Isolated Rabbit Tibialis Anterior Muscle. Journal of Biomechanics, 36, 505-512. http://dx.doi.org/10.1016/S0021-9290(02)00430-X

[47] Maloiy, G.M.O., Alexander, R.McN., Njau, R. and Jayes, A.S. (1979) Allometry of Legs of Running Birds. Journal of Zoology (London), 187, 161-167. http://dx.doi.org/10.1111/j.1469-7998.1979.tb03940.x

[48] Medler, S. (2002) Comparative Trends in Shortening Velocity and Force Production in Skeletal Muscles. American Journal of Physiology-Regulatory Integrative and Comparative Physiology, 283, R368-R378.

[49] Seow, C.Y. and Ford, L.E. (1991) Shortening Velocity and Power Output of Skinned Muscle Fibres from Mammals Having a 25,000-Fold Range of Body Mass. Journal of General Physiology, 97, 541-560. http://dx.doi.org/10.1085/igp.97.3.541

[50] Schilder, J.R. and Marden, J.H. (2004) A Hierarchical Analysis of the Scaling of Force and Power Production by Dragonfly Flight Motors. Journal of Experimental Biology, 207, 767-776. http://dx.doi.org/10.1242/jeb.00817

[51] Mendez, J. and Keys, A. (1960) Density and Composition of Mammalian Muscle. Metabolism, 9, 184-188. 


\section{Appendix A. List of Notations}

Abbreviations

PCSA - physiologic cross-sectional area

Mathematical signs and symbols

$=$ - common equality sign

$\equiv$-identity sign implying "by definition"

$\approx$-approximate equality sign

$\sim$-proportionality relation symbol omitting only numerical coefficients

$\cong$-here used as similarity sign supporting only physical dimension units

$\propto$-here used as scaling rule symbol not supporting dimension units

Physical and geometrical notations

$\alpha_{m}$-muscle-mass allometric index

$\varepsilon_{m}^{(\text {opt })}$ - muscle strain in the optimum-velocity dynamic regime

$\eta_{m}$ - muscle structure parameter

$\rho_{\text {tiss }}$ - tissue density

$\rho_{m}$ - muscle density

$\sigma_{\text {tiss }}^{(\max )}$ - peak tissue stress

$\Delta L$-length change

$\Delta F$-force change

$\Delta t_{m}$-activation timing of muscle $m$

$A_{r m}$ - cross-sectional area of muscle $m$ in passive $(r=0)$ and active $(r \neq 0)$ states

$a$-scaling exponent for cross-sectional area

$D$-diameter of ideal cylinder

$E_{r m}$-active-muscle elastic modulus establishing the dynamic regime $r \neq 0$

$e$-strain scaling exponent

$\Delta F_{\text {elast }}^{(\max )}=\Delta F_{m}^{(\max )}$-maximum active elastic force

$F_{\text {prod }}^{(\text {fast })}$ - production force by fast muscle

$F_{\text {motor }}^{\text {(conc) }}$-elastic force adapted to concentric work in motor muscle

$F_{\text {musc }}^{(\max )}$-maximum force exerted by muscle

$K_{m}$ - active muscle stiffness

$K_{s}$ - sarcomere/cellular stiffness

$L$ - length of an ideal cylinder

$L_{m}$ - variable muscle length in non-specified dynamics

$L_{r m}$ - dynamic muscle length in the regime $r$

$l$-length exponent

$m$-as index, muscle in unspecified function

$M$-body mass of animals

$M_{m}$-muscle mass

$r$-numerical parameter indicating transient dynamic states via optimum-velocity $(r=1)$, moderate-velocity

$(r=2)$, and high-velocity $(r=3)$ dynamic regimes, distinct of passive muscle state $(r=0)$.

$T_{r m}$ - period of cycling in the adapted regime $r$

$V_{r m}$ - muscle contraction velocity in the dynamic regime $r$

W - body weight

\section{Appendix B. Scaling Muscle Functions}

The motor function or motor activity is associated with the generation of the active force of amplitude $F_{\text {prod }}^{(\max )}$ at moderate contraction velocities during muscle shortening, below the turning points 2 in the insets a, b and c in Figure 1. In fast-fibre muscles, the corresponding concentric force 


$$
F_{\text {motor }}^{(\text {conc })}=F_{\text {elast }}^{(\max )}=\Delta F_{2 m}^{(\text {conc })} \sim E_{2 m}^{(\text {fast })} A_{2 m}^{3 / 2} L_{2 m}^{-1} \cong F_{\text {prod }}^{(\text {fast })}
$$

is described by the known universal pattern of the maximal elastic forces [37] emerging during pure bending, pure torsion, as well as complex bending-torsion loading of a long cylinder of length $L_{2 m}$ and cross-sectional area $A_{2 m}$ [30]. The exploration of Equation (35) though Equation (8), Equation (16), Equation (24), and Equation (26) results in the fast-muscle-force constraint $3 a_{m} / 2-l_{m}=1+\alpha_{m}$. It is remarkable that the case of slow-fibre muscle, namely

$$
F_{\text {motor }}^{(\text {conc) }}=F_{\text {elast }}^{(\max )}=\Delta F_{2 m}^{(\text {conc })} \sim E_{2 m}^{(\text {slow })} A_{2 m}^{3 / 2} L_{2 m}^{-1} \cong F_{\text {prod }}^{(\text {slow })}
$$

results in the slow-muscle-force constraint $3 a_{m} / 2-2 l_{m}=a_{m}$, which is exactly the same as fast muscle, in view of function-independent Equation (10). Therefore, any muscle tuned to the motor locomotor function should expose its dynamic structure scaled by

$$
a_{\text {motor }}^{(\text {conc })}=\frac{4}{5}\left(1+\alpha_{\text {motor }}\right), l_{\text {motor }}^{(\text {conc })}=\frac{1}{5}\left(1+\alpha_{\text {motor }}\right),
$$

regardless of the fibre type content. This finding follows from both the muscle-force constraints solved with the help of the function-independent muscle-shape constraint shown in Equation (10). Moreover, as shown in [30], the principal component of the compressive stress $\sigma_{m}^{(\text {conc) }}$, specified in Equation (17), may be caused by the peak transverse-tensile strains

$$
\varepsilon_{\text {motor }}^{(\text {conc })}=\frac{\Delta D_{m}^{(\max )}}{L_{m}} \propto M_{m}^{e_{m}}, \text { with } e_{m}=e_{\text {motor }}^{(\text {conc })}=\frac{a_{m}}{2}-l_{m},
$$

where $\Delta D_{m}^{(\max )} \sim D_{m} \sim A_{m}^{1 / 2}$ is transverse muscle deformation.

Likewise, the maximum elastic eccentric force

$$
F_{\text {brake }}^{(\text {eccen })}=\Delta F_{2 m}^{(\text {eccen })} \sim E_{2 m}^{(\text {eccen })} A_{2 m}^{2} L_{2 m}^{-2} \cong F_{\text {prod }}^{(\text {fast })},
$$

associated with the brake muscle function (see the inset $\mathrm{b}$ in Figure 1) provides the maximum elastic stress

$$
\sigma_{\text {brake }}^{(\max )}=\frac{F_{\text {brake }}^{(\text {eccen }}}{A_{m}} \propto M_{m}^{s_{m}} \text {, with } s_{m}=s_{\text {brake }}^{(\text {eccen })}=a_{m}-2 l_{m},
$$

following from Equation (17) and Equation (39). The unique solution to both fast-muscle-force constraint, $2 a_{m}-2 l_{m}=1+\alpha_{m}$, and slow-muscle-force constraint, $2 a_{m}-3 l_{m}=a_{m}$, is

$$
a_{\text {brake }}^{(\text {eccen })}=\frac{3}{4}\left(1+\alpha_{\text {brake }}\right), l_{\text {brake }}^{(\text {eccen })}=s_{\text {brake }}^{(\text {eccen })}=\frac{1}{4}\left(1+\alpha_{\text {brake }}\right) \text {. }
$$

The strut muscle function treated as antagonistic to both motor and brake functions drives nearly isometric contractions characteristic of small, but non-zero length change $\left(\Delta L_{m} \ll L_{m}\right)$ achieved near peak forces (see the inset $\mathrm{c}$ in Figure 1). This suggests the isometric force

$$
F_{\text {strut }}^{(\text {isom })}=E_{2 m}^{(\text {isom })} \varepsilon_{2 m}^{(\text {isom })} A_{2 m} \cong F_{\text {prod }}^{(\text {fast })} \text {, with } \varepsilon_{2 m}^{(i s o m)}=\Delta L_{2 m}^{(i s o m)} / L_{2 m},
$$

produce by fast muscles. Again, one solves the muscle strut constraints $2 a_{m}+l_{m}=1+\alpha_{m}$ and $2 a_{m}+2 l_{m}=a_{m}$ resulting in

$$
a_{\text {strut }}^{(\text {isom })}=1+\alpha_{\text {strut }} \text { and } l_{\text {strut }}^{(\text {isom })}=s_{\text {strut }}^{(\text {isom })}=0 \text {, with } \Delta L_{2 m}^{(i s o m)} \propto L_{2 m}^{2} \text {, }
$$

for any type of muscles.

A new antagonist (to strut muscle) tuned to the cardiac type contractions via active elastic force

$$
F_{\text {pump }}^{(\text {card })}=\Delta F_{2 m}^{(\text {card })} \sim E_{2 m}^{(\text {card })} L_{2 m}^{2} \cong F_{\text {prod }}^{(\text {fast })}
$$

is associated with, say, pump function providing the fast-muscle-force constraint $2 l_{m}=1+\alpha_{m}$. This yields

$$
a_{\text {pump }}^{(\mathrm{card})}=l_{\text {pump }}^{(\mathrm{card})}=s_{\text {pump }}^{(\mathrm{card})}=e_{\text {pump }}^{(\mathrm{card})}=\frac{1}{2}\left(1+\alpha_{\text {pump }}\right),
$$


equally applied to slow-fibre muscles resulting in the slow-force constraint $l_{m}=a_{m}$.

To complete the intrinsic-force description, the spring-type control function associated with the elastic force

$$
F_{\text {contr }}^{(\text {sprin }}=F_{\text {elast }}^{(\text {opt })} \propto E_{1 m}^{(\text {slow })} M_{m}^{2 / 3} \propto E_{1 m}^{(\text {slow })} A_{m}^{2 / 3} L_{m}^{2 / 3} \cong F_{\text {prod }}^{(\text {slow }},
$$

produced by slow-fibre muscles, results in

$$
a_{\text {cont }}^{(\text {sprin })}=\frac{2}{3}\left(1+\alpha_{\text {cont }}\right), l_{\text {cont }}^{(\text {sprin })}=\frac{1}{3}\left(1+\alpha_{\text {cont }}\right) \text {, with } s_{\text {cont }}^{(\text {sprin })}=e_{\text {cont }}^{(\text {sprin })}=0,
$$

This solution follows from the slow-force and fast-force constraints $2\left(a_{m}+l_{m}\right) / 3=a_{m}$ and $\left(2 a_{m}+5 l_{m}\right) / 3=1+\alpha_{m}$ and therefore is valid for any type of multifunctional muscle [13] tuned to the optimum-velocity regime. All obtained specific-function mechanical characteristics are summarized in Table 2. 
Scientific Research Publishing (SCIRP) is one of the largest Open Access journal publishers. It is currently publishing more than 200 open access, online, peer-reviewed journals covering a wide range of academic disciplines. SCIRP serves the worldwide academic communities and contributes to the progress and application of science with its publication.

Other selected journals from SCIRP are listed as below. Submit your manuscript to us via either submit@scirp.org or Online Submission Portal.
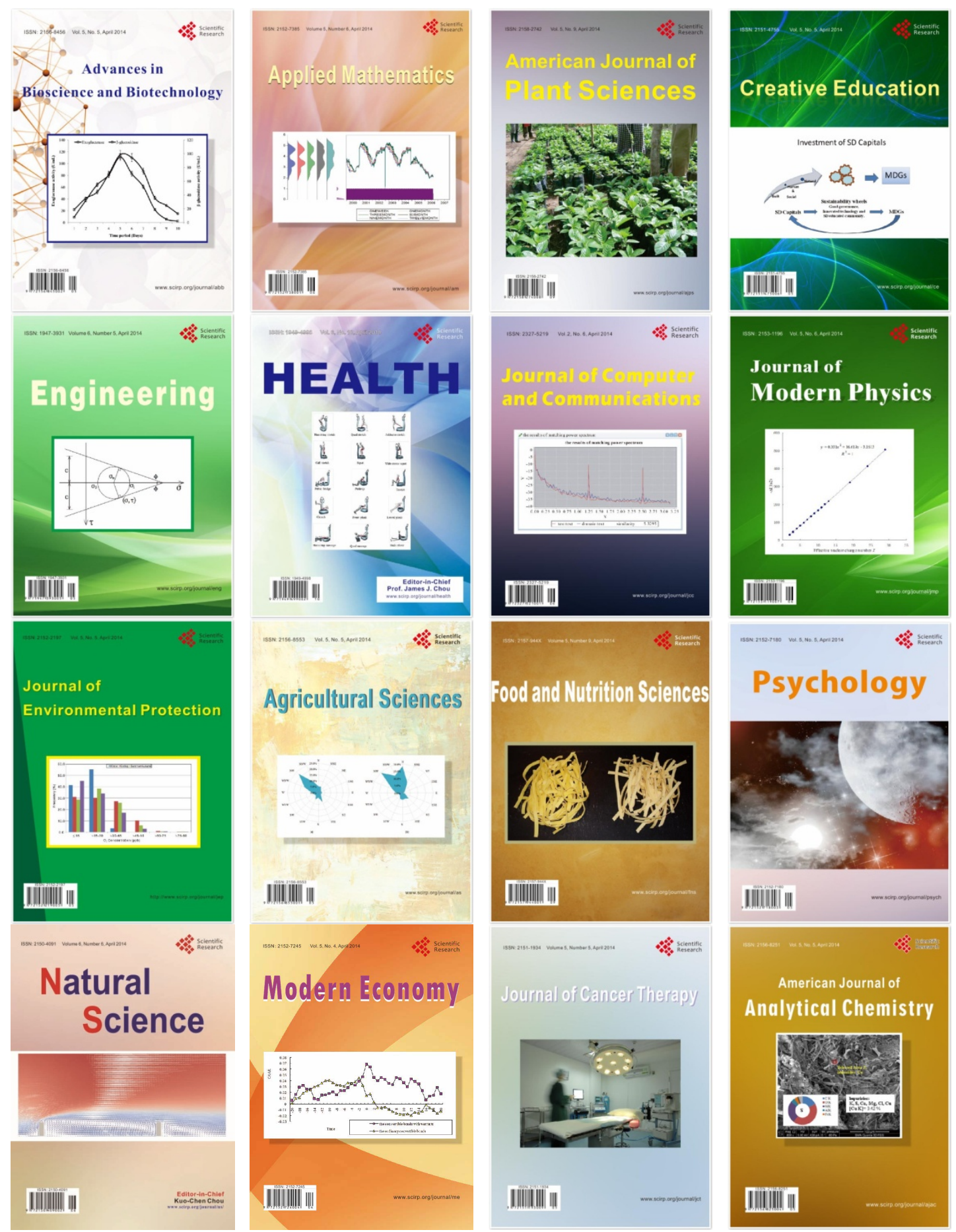